\title{
Least costly identification experiment for the identification of one module in a dynamic network
}

\author{
F. Morelli ${ }^{1}$, X. Bombois ${ }^{1,2}$, H. Hjalmarsson ${ }^{3}$, L. Bako ${ }^{1}$, and K. Colin ${ }^{1}$ \\ ${ }^{1}$ Laboratoire Ampère, Ecole Centrale de Lyon, Université de Lyon, Ecully, France \\ ${ }^{2}$ Centre National de la Recherche Scientifique (CNRS), France \\ ${ }^{3}$ Automatic Control, School of Electrical Engineering, KTH, Stockholm, Sweden
}

November 19, 2019

\begin{abstract}
In this paper we consider the design of the least costly experiment for the identification of one module in a given network of locally controlled systems. The identification experiment will be designed in such a way that we obtain a sufficiently accurate model of the to-be-identified module with the smallest identification cost i.e. with the least perturbation of the network.
\end{abstract}

\section{Introduction}

This paper contributes to the efforts of developing techniques for the identification of large-scale or interconnected systems when the topology of the network is known. In many papers, the problem is seen as a multivariable identification problem and structural properties of the system are then used to simplify this complex problem (see e.g. [15]). The identifiability of the multivariable structure is studied in a prediction error context in [32] while this multivariable structure is exploited in other papers to reduce the variance of a given module in the network (see $[16,14,6]$ ). In other contributions, conditions are derived for the consistent estimation of a given module in a dynamic network (see e.g. $[5,12,18]$ ).

While many different problems have thus been extensively studied in the dynamical network context, this is not the case for optimal experiment design (i.e. the problem of designing the excitation signal of an identification experiment to guarantee a certain model accuracy under some constraints on this excitation signal). In our previous contribution [2], we made the first steps towards optimal experiment design in a dynamical network context. In [2], we considered the case of a network made up of locally controlled systems, i.e. modules, whose interconnection is realized by exchanging their measured output between neighbouring modules (this type of networks is usual in the literature on multi-agent systems (see e.g. $[9,19]$ )). For this particular type of dynamical networks, we showed how to design the excitation signals that have to be added to each module in order to identify models of these different modules that are sufficiently accurate to enhance the network performance by a redesign of the local controllers. The accuracy of each model can be measured by the inverse of the covariance matrix of the identified parameter vector of each module. In [2], we have derived an expression for the inverse of this covariance matrix as an affine function of the excitation signal spectra. It is important to note that the inverse of the covariance matrix of a given module $l$ is obviously a function of the excitation signal applied to this particular module, but also, though in a lesser extent, a function of the excitation signals applied to all modules $k$ 
having a path to $l$. Consequently, the excitation signal applied to such a module $k$ contributes to the accuracy of the model of $l$. In other words, the propagation of the excitation signals due to the interconnection is a positive feature when we want to obtain sufficiently accurate estimates of every module with the smallest excitation power (see also $[31,7,8]$ ).

In some cases, we may only be interested in the accurate identification of one specific module $l$ via the application of an excitation signal to $l$. Since the other modules have not to be identified, the propagation of this excitation signal to the other modules due to the interconnection is a negative feature that have to be limited as much as possible ${ }^{1}$. For this purpose, we extend the least costly identification framework (see [4]) to this particular dynamic network identification problem. In particular, we design the spectrum of the excitation signal applied to $l$ in such a way that the accuracy of the identified model (measured via the inverse of the covariance matrix) is larger than a given threshold while entailing the smallest perturbation on the network. The perturbation (i.e. the cost of the identification) will be measured by the sum of the effects of the excitation signal on the input and output of each system in the network.

With respect to the least costly framework introduced in [4] for a single closed loop, the cost of the identification experiment in the network context thus not only contains the perturbation induced by the excitation signal in the closed loop where the system has to be identified, but also the perturbation induced in other loops by this excitation signal. This propagation of the effect of the excitation signal is due to the fact that the output signal of the to-be-identified loop (which is perturbed by the excitation signal) is transmitted to neighbouring modules. In this paper, in order to reduce this propagation, we propose an approach where the signal transmitted to the neighbouring modules is no longer the actual output signal, but a sanitized version of this output signal where the contribution of the excitation signal has been (partially) removed. Indeed, using an initial estimate of the to-be-identified system, we derive an estimate of this contribution and we subtract this estimate from the measured output signal before the transmission to the neighbouring modules.

This new configuration is inspired by the concept of stealth identification that we introduced in [26] for a single closed loop and that we here extend to the network case. The use of the stealth identification in this paper is also a new application of this concept since, in [26], it was introduced as a tool to enable classical optimal experiment design in a loop where the controller is not Linear Time Invariant (LTI). With respect to $[26]^{2}$, we also analyze which accuracy condition the initial estimate used to compute the sanitized version of the output signal must respect for the stealth configuration to be effective (i.e., to yield a smaller identification cost). Moreover, another contribution of the present paper is to robustify the stealth approach by considering the uncertainty of this initial estimate and its influence on the cost of the identification. For this purpose, as we will see in the sequel, we will need to consider an optimal experiment design where the cost constraint is robustified with respect to the initial uncertainty of the initial estimate. In [3], we have recentlty proposed an approach to tackle such a robustified cost constraint. With respect to the earlier approaches for this problem [27, 20, 10], the approach in [3] does not entail any kind of approximation. However, the possible high dimension of the network can imply an excessive computational complexity for the approach in [3]. To avoid this problem, we propose in this paper an alternative approach which is more appropriate to the network situation (and its possible high dimension) and which, while being (slightly) more conservative, has the same property as the one in [3] i.e., it is not based on any approximation. This absence of approximation is also the main difference with the approach proposed in the conference paper [24] on which the present paper is

\footnotetext{
${ }^{1}$ This is especially the case when the experiment is performed in a network where all modules have to track a given reference. In this case, the excitation signal introduces an undesired perturbation on the tracking performance.

${ }^{2}$ and with respect to the conference paper [24] on which the present paper is based
} 
based.

Note finally that the framework considered here is much different than the frameworks of [30, 17], which are, to our knowledge, the only other papers treating the optimal experiment design problem in a network. In [30], the authors consider input design for nonparametric identification of static nonlinearities embedded in a network. The main purpose of [17] lies in the use of measurable disturbances in optimal experiment design.

Notations: The matrix $I_{n}$ denotes the identity matrix of dimension $n$. The matrix

$$
\left(\begin{array}{ccc}
X_{1} & 0 & 0 \\
0 & \ddots & 0 \\
0 & 0 & X_{N}
\end{array}\right)
$$

will be denoted $\operatorname{diag}\left(X_{1}, \ldots, X_{N}\right)$ when the elements $X_{i}(i=1, \ldots, N)$ are scalar quantities, while it will be denoted $\operatorname{bdiag}\left(X_{1}, \ldots, X_{N}\right)$ when the elements $X_{i}(i=1, \ldots, N)$ are matrices. For a matrix $A, A^{T}$ denotes the transpose of $A$ and $A^{*}$ its conjugate transpose. For a vector of transfer function $R(z), R_{i}(z)$ denotes the $i^{\text {th }}$ entry of $R(z)$. Finally, $\otimes$ denotes the Kronecker product and $\star$ the Redheffer star product [33].

\section{Description of the network configuration}

We consider a network made up of $N_{\text {mod }}$ single-input single-output (SISO) systems $\mathcal{S}_{i}\left(i=1, \ldots, N_{\text {mod }}\right)$ operated in closed loop with a SISO decentralized controller $K_{i}\left(i=1, \ldots, N_{\text {mod }}\right)$ :

$$
\begin{array}{r}
\mathcal{S}_{i}: y_{i}(t)=G_{i}\left(z, \theta_{0, i}\right) u_{i}(t)+v_{i}(t) \\
u_{i}(t)=K_{i}(z)\left(y_{r e f, i}(t)-y_{i}(t)\right)
\end{array}
$$

where the signal $u_{i}$ is the input applied to the system $\mathcal{S}_{i}$ and $y_{i}$ is the measured output. This output is made up of a contribution of the input $u_{i}$ and of a disturbance term $v_{i}(t)=H_{i}\left(z, \theta_{0, i}\right) e_{i}(t)$ that represents both process and measurement noises. The different systems $\mathcal{S}_{i}\left(i=1, \ldots, N_{\text {mod }}\right)$ are thus described by two stable transfer functions $G_{0, i}(z)=G_{i}\left(z, \theta_{0, i}\right)$ and $H_{0, i}(z)=H_{i}\left(z, \theta_{0, i}\right)$, the later being also minimum-phase and monic. These transfer functions are parametrized by an unknown true parameter vector $\theta_{0, i} \in \mathbf{R}^{n_{\theta_{i}}}$ in a known model structure. For each $i(i=$ $\left.1, \ldots, N_{\text {mod }}\right)$, the signal $e_{i}\left(i=1, \ldots, N_{\text {mod }}\right)$ defining $v_{i}$ is a zero mean white noise signal of variance $\sigma_{e_{i}}^{2}$ and these white noise signals $e_{i}$ are for simplicity supposed to be mutually independent. In (2), $y_{r e f, i}$ is a reference signal that will be computed based on the measured outputs of neighbouring modules (see later). We can rewrite the above equations as follows:

$$
\begin{array}{r}
\bar{y}(t)=\bar{G}\left(z, \theta_{0}\right) \bar{u}(t)+\bar{H}\left(z, \theta_{0}\right) \bar{e}(t) \\
\bar{u}(t)=\bar{K}(z)\left(\bar{y}_{r e f}(t)-\bar{y}(t)\right)
\end{array}
$$

where $\bar{y}=\left(y_{1}, \ldots, y_{N_{\text {mod }}}\right)^{T}$ and $\bar{u}, \bar{e}, \bar{y}_{\text {ref }}$ are defined in a similar way and where $\theta_{0}=\left(\theta_{0,1}^{T}, \ldots, \theta_{0, N_{\text {mod }}}^{T}\right)^{T}$ $\in \mathbf{R}^{n_{\theta}}$ concatenates the true parameter vectors $\theta_{0, i}\left(i=1, \ldots, N_{\text {mod }}\right)$. In these equations, we also use the notation $\bar{G}=\operatorname{diag}\left(G_{1}, \ldots, G_{N_{\text {mod }}}\right)(\bar{H}$ and $\bar{K}$ are defined in a similar way).

The closed-loop systems described in (3)-(4) will be interconnected via the following equation:

$$
\bar{y}_{\text {ref }}(t)=\mathcal{A} \bar{y}(t)+\mathcal{B} r e f_{\text {ext }}(t)
$$

where the matrix $\mathcal{A}$ and the vector $\mathcal{B}$ represent the flow of information in the network and $r e f_{\text {ext }}$ is a (scalar) external reference signal that should be followed by all outputs $y_{i}$ and that is generally 
only available at one node of the network. This type of interconnections is typical in formation control or multi-agent systems (see e.g. $[9,19]$ ).

To illustrate (5), let us consider the network represented in Figure 1. In this network, we have $N_{\text {mod }}=6$ systems/modules, all of the form (1) and all operated as in (2) with a decentralized controller $K_{i}$ (see Figure 2). These local closed loops are represented by a circle/node in Figure 1. The objective of this network is that the outputs $y_{i}$ of all modules follow the external reference $r e f_{\text {ext }}$ even though this reference is only available at Node 1. For this purpose, a number of nodes are allowed to exchange information (i.e. their measured output) with some other neighbouring nodes. The arrows between the nodes in Figure 1 indicate the flow of information.

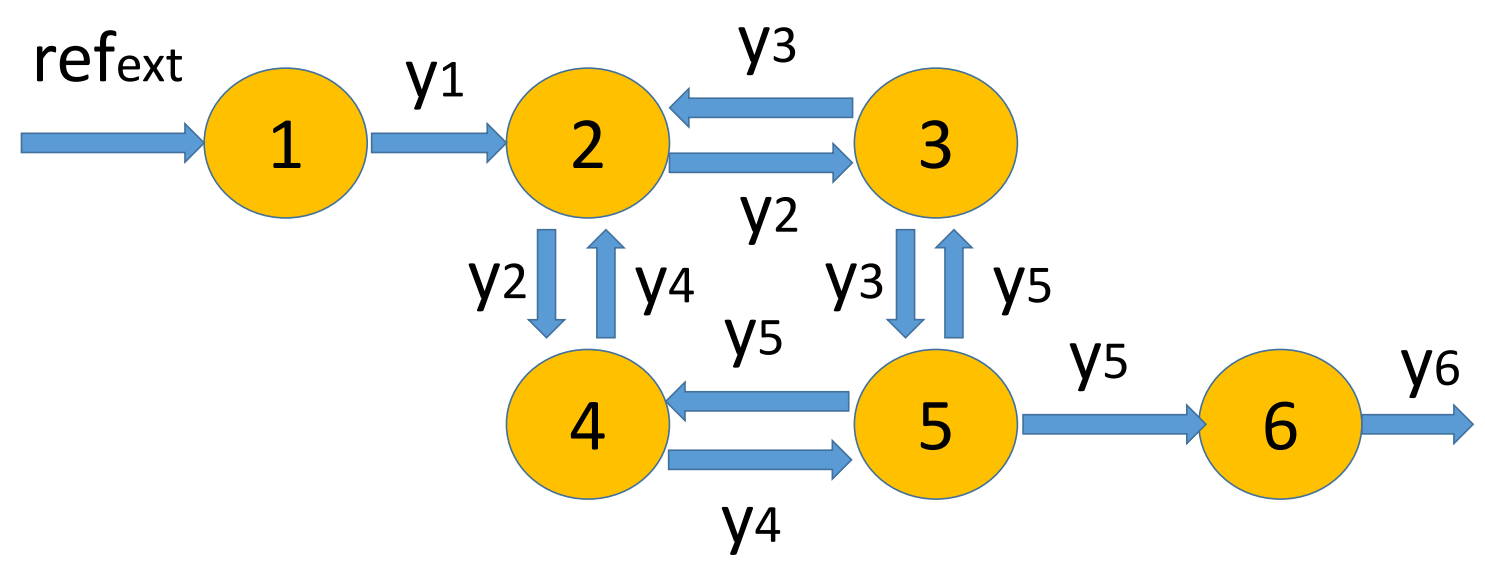

Figure 1: Example of graph representation of the network, each circle represents a node $i$ and the edges represent the communication link between the nodes

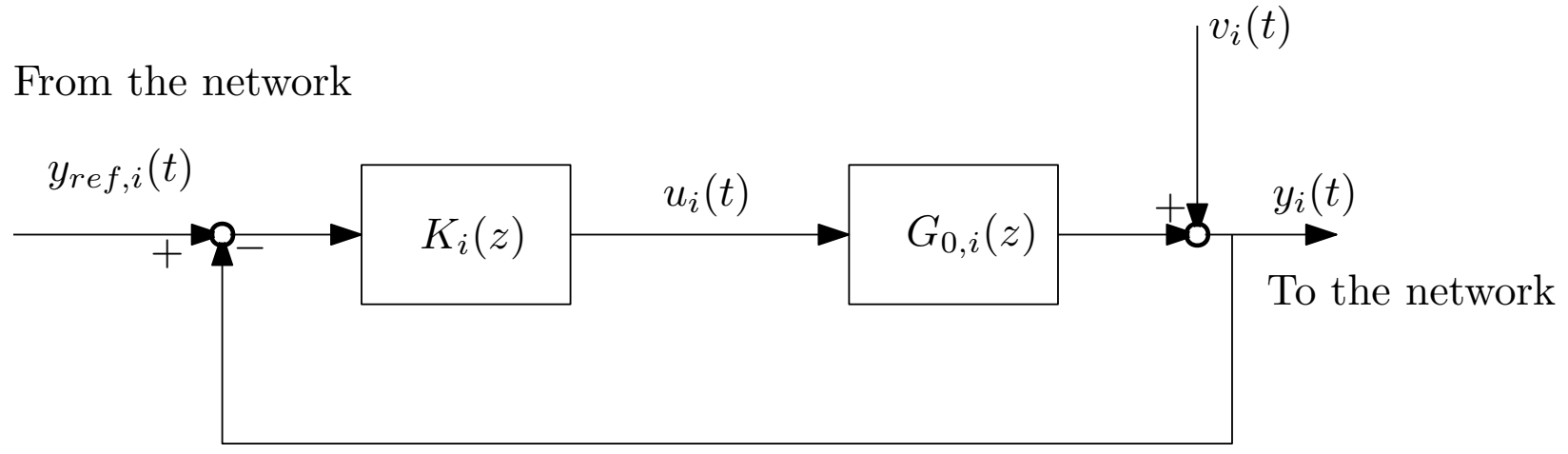

Figure 2: Representation of a single module/node $i$

For example, Node 5 receives the output of two nodes (i.e. Nodes 3 and 4 ) and sends its output (i.e. $y_{5}$ ) to three nodes (Nodes 3, 4 and 6). The reference signal $y_{r e f, i}$ of Node $i$ will be computed as a linear combination of the received information at Node $i$. For Node 5, $y_{r e f, 5}$ will thus be a 
linear combination of $y_{3}$ and $y_{4}$. More precisely, for all outputs $y_{i}$ to be able to follow the external reference $r e f_{\text {ext }}, \mathcal{A}$ and $\mathcal{B}$ in (5) are generally chosen as $[9,19]$ :

$$
\mathcal{A}=\left(\begin{array}{cccccc}
0 & 0 & 0 & 0 & 0 & 0 \\
1 / 3 & 0 & 1 / 3 & 1 / 3 & 0 & 0 \\
0 & 0.5 & 0 & 0 & 0.5 & 0 \\
0 & 0.5 & 0 & 0 & 0.5 & 0 \\
0 & 0 & 0.5 & 0.5 & 0 & 0 \\
0 & 0 & 0 & 0 & 1 & 0
\end{array}\right) \quad \mathcal{B}=(1,0, \ldots, 0)^{T}
$$

The matrix $\mathcal{A}$ is called the normalized adjacency matrix in the literature [9]. Using (5), we, e.g., see that the tracking error signals $y_{r e f, 1}-y_{1}$ and $y_{r e f, 2}-y_{2}$ of Nodes 1 and 2 are respectively given by refext $-y_{1}$ and $1 / 3\left(\left(y_{1}-y_{2}\right)+\left(y_{3}-y_{2}\right)+\left(y_{4}-y_{2}\right)\right)$. Similar relations can be found for all the other nodes. If the different loops $\left[K_{i} G_{i}\right]$ are designed to make the tracking error $y_{r e f, i}-y_{i}$ as small as possible, it can be proven that such an interconnection allows good tracking of $r e f_{\text {ext }}$ at all nodes $[19,9]$. A normalized adjacency matrix can be defined for any information flow using the following rules. Row $i$ of $\mathcal{A}$ is zero if no output is sent to Node $i$. If $y_{i}$ is sent to Node $j$, entry $(j, i)$ of $\mathcal{A}$ will be nonzero. Finally, all nonzero entries in a row are equal and sum up to one.

We also need to introduce the notion of (directed) path between two nodes. There exists a path from Node $i$ to Node $j$ if $\mathcal{A}_{j i} \neq 0$ or we can find a set of $\zeta$ intermediary nodes described by the indexes $\left\{n_{1}, \ldots, n_{\zeta}\right\}$ such that $\mathcal{A}_{n_{1} i} \neq 0, \mathcal{A}_{n_{2} n_{1}} \neq 0, \ldots, \mathcal{A}_{j n_{\zeta}} \neq 0$. We moreover introduce the following definitions:

Definition 1 Consider an arbitrary node of a network containing $N_{\text {mod }}$ nodes, say Node $j(j=$ $\left.1, \ldots, N_{\text {mod }}\right)$. For this node, we define the set $\mathcal{P}_{j}$ as a set of indexes of nodes. A certain index $i \neq j$ belongs to $\mathcal{P}_{j}$ if there exists a path from Node $j$ to Node $i$. Similarly, for the same Node $j$, we also define the set $\mathcal{L}_{j}$. A certain index $i \neq j$ belongs to $\mathcal{L}_{j}$ if there exists a path from Node $i$ to Node $j$.

As an example, $\mathcal{P}_{5}=\{2,3,4,6\}$ and $\mathcal{L}_{5}=\{1,2,3,4\}$ for the network of Figure 1. For the sequel, it is important to note the following facts. If an external signal (e.g., the disturbance $v_{i}$ or an excitation signal $r$ ) is added to Node $j$, this external signal will also influence all nodes $i$ with $i \in \mathcal{P}_{j}$. Conversely, Node $j$ will be influenced by all external signals added in nodes $i$ with $i \in \mathcal{L}_{j}$.

In the sequel, we will suppose that an identification procedure has delivered initial estimates $\theta_{\text {init }, i}$ of $\theta_{0, i}\left(i=1, \ldots, N_{\text {mod }}\right)$ and that all these estimates are normally distributed around $\theta_{0, i}$ with a covariance matrix $P_{\text {init }, i}$. This can e.g. be done using an open-loop experiment on each system $\mathcal{S}_{i}$ disconnected from the network or via the identification procedure presented in [2] (the experiment is then done in the network configuration). We can then say that the concatenated vector $\theta_{\text {init }}=\left(\theta_{\text {init }, 1}^{T}, \ldots, \theta_{\text {init }, N_{\text {mod }}}^{T}\right)^{T}$ is normally distributed around $\theta_{0}=\left(\theta_{0,1}^{T}, \ldots, \theta_{0, N_{\text {mod }}}^{T}\right)^{T}$ with a covariance matrix $P_{\text {init }}=\operatorname{bdiag}\left(P_{\text {init }, 1}, \ldots, P_{\text {init }, N_{\text {mod }}}\right)$. Based on this statistical property, the following ellipsoid $U_{\text {init }}$ is a $\beta \%$-confidence region for the unknown parameter vector $\theta_{0}$ :

$$
U_{\text {init }}:=\left\{\theta \in \mathbf{R}^{n_{\theta}} \mid\left(\theta-\theta_{\text {init }}\right)^{T} P_{\text {init }}^{-1}\left(\theta-\theta_{\text {init }}\right)<\chi_{\beta}\right\}
$$

where $\operatorname{Pr}\left(\chi^{2}\left(n_{\theta}\right)<\chi_{\beta}\right)=\beta$ and $\theta=\left(\theta_{1}^{T}, \ldots, \theta_{N_{m o d}}^{T}\right)^{T}$. This ellipsoid $U_{\text {init }}$ can be considered as an uncertainty set for the unknown true parameter vector $\theta_{0}$. From now onwards, we will therefore assume that $\theta_{0} \in U_{\text {init }}$.

In the sequel, we will suppose that the model accuracy obtained after this initial experiment is satisfactory for all, but one node, say Node $l\left(l=1, \ldots, N_{\text {mod }}\right)$. We will therefore have to perform a new identification experiment to obtain a better estimate of the parameter vector $\theta_{0, l}$ describing this node. This experiment is described in the next section. 


\section{Identification of one module in the network and cost of the experiment}

\subsection{Cost of an experiment in the stealth and non-stealth configurations}

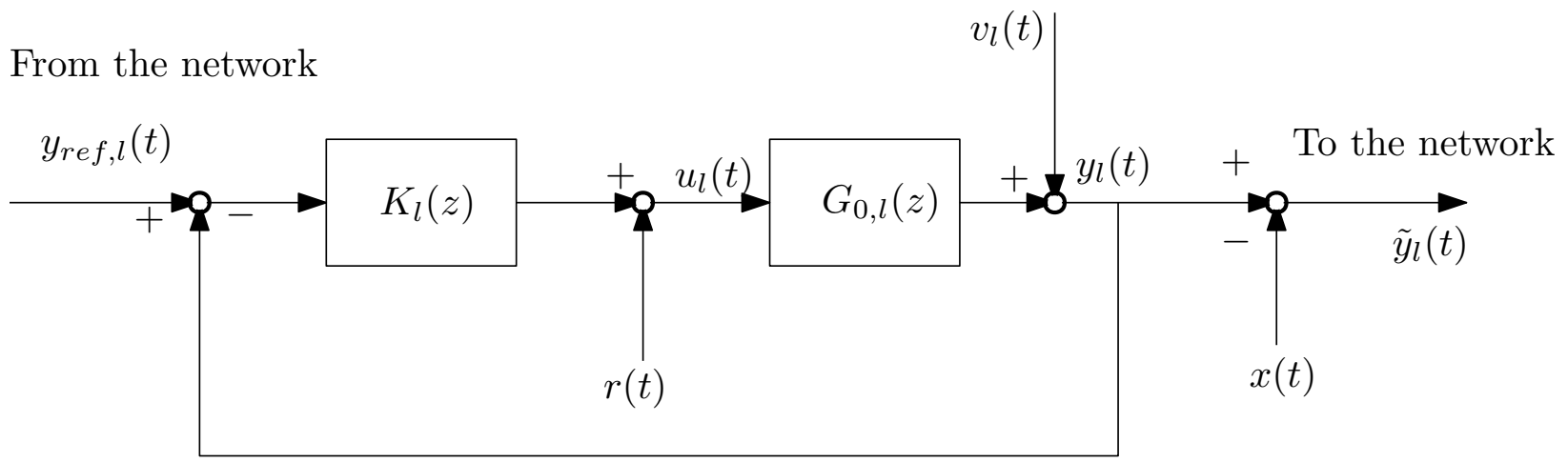

Figure 3: To-be-identified node (i.e. Node $l$ ) during the identification experiment. In the stealth setting, $x(t)$ is given by (7). In the non-stealth setting, $x(t)=0$.

During the identification experiment, we will apply for a duration $N$ (i.e. from $t=1$ till $t=N$ ) an excitation signal $r(t)$ of spectrum $\Phi_{r}$ at the output of the controller $K_{l}$ of Node $l$ (see Figure 3 ). This excitation will allow to obtain sufficiently informative data $u_{l}(t)$ and $y_{l}(t)(t=1, \ldots, N)$ for an accurate identification of $\mathcal{S}_{l}$ (see Section 3.2). In Figure 3, we also observe the signal $x(t)$. In this paper, we will consider two choices for $x(t)$ corresponding to two configurations/settings: the stealth and the non-stealth configurations. In the non-stealth setting, the signal $x(t)$ will be chosen equal to zero. This choice corresponds to the classical setting for an identification experiment in a closed-loop/network context (see e.g. [2]). In the stealth setting, $x(t)$ will be chosen as the following estimate of the contribution of $r$ in $y_{l}$ :

$$
x(t)=\underbrace{\frac{G_{l}\left(z, \theta_{\text {init }, l}\right)}{1+K_{l}(z) G_{l}\left(z, \theta_{\text {init }, l}\right)}}_{=T_{\text {init }, l(z)}} r(t) \quad(t=1, \ldots, N)
$$

where $\theta_{\text {init, },}$ is the subvector of $\theta_{\text {init }}$ corrresponding to $\mathcal{S}_{l}$. Note that $(7)$ can be easily computed since both $r$ and $T_{\text {init }, l}(z)$ are known.

As shown in Figure 3, the signal $x(t)$ is subtracted from the measured output $y_{l}(t)$ to give $\tilde{y}_{l}(t)=y_{l}(t)-x(t)$ which will be the signal that will be transmitted to compute $\bar{y}_{\text {ref }}$. Consequently, during the identification experiment, the equations (4)-(5) become:

$$
\begin{aligned}
\bar{u}(t) & =\bar{m}_{l} r(t)+\bar{K}(z)\left(\bar{y}_{r e f}(t)-\bar{y}(t)\right) \\
\bar{y}_{r e f}(t) & =\mathcal{A}\left(\bar{y}(t)-\bar{m}_{l} x(t)\right)+\mathcal{B} r e f_{\text {ext }}(t)
\end{aligned}
$$

where $\bar{m}_{i}\left(i=1, \ldots, N_{\text {mod }}\right)$ denotes a unit (column) vector of dimension $N_{\text {mod }}$ for which the $i^{t h}$ entry is equal to 1 and the other entries are equal to zero.

We will show, in the sequel, the advantage of the stealth setting in order to reduce the cost of the identification experiment. For this purpose, we need to define the latter. Let us first consider the stealth setting. In this case, the output vector $\bar{y}$ and the input vector $\bar{u}$ in the network configuration (3)-(8)-(9) can be rewritten as follows as a function of the external signals $r$, re $f_{\text {ext }}$ and $\bar{e}$ : 


$$
\begin{aligned}
& \bar{y}(t)=R^{y}\left(z, \theta_{0}\right) r(t)+R_{\text {ext }}^{y}\left(z, \theta_{0}\right) r e f_{\text {ext }}(t)+S^{y}\left(z, \theta_{0}\right) \bar{e}(t) \\
& \bar{u}(t)=R^{u}\left(z, \theta_{0}\right) r(t)+R_{\text {ext }}^{u}\left(z, \theta_{0}\right) r e f_{\text {ext }}(t)+S^{u}\left(z, \theta_{0}\right) \bar{e}(t)
\end{aligned}
$$

for some vectors of transfer functions $R_{e x t}^{y}, R_{e x t}^{u}$, some matrices of transfer functions $S^{y}, S^{u}$ and

$$
\begin{aligned}
& R^{u}\left(z, \theta_{0}\right)=\bar{m}_{l} S_{0, l}(z)+\overline{\mathcal{N}}\left(z, \theta_{0}\right)\left(T_{0, l}(z)-T_{\text {init }, l}(z)\right) \\
& R^{y}\left(z, \theta_{0}\right)=\bar{G}\left(z, \theta_{0}\right) R^{u}\left(z, \theta_{0}\right)
\end{aligned}
$$

where $S_{0, l}(z)=1 /\left(1+K_{l}(z) G_{l}\left(z, \theta_{0, l}\right)\right)$ and $T_{0, l}(z)=G_{l}\left(z, \theta_{0, l}\right) /\left(1+K_{l}(z) G_{l}\left(z, \theta_{0, l}\right)\right)$ are scalar transfer functions and $\overline{\mathcal{N}}\left(z, \theta_{0}\right)$ is a vector of transfer functions:

$$
\begin{aligned}
& \overline{\mathcal{N}}\left(z, \theta_{0}\right)=\bar{K}(z) \bar{S}\left(z, \theta_{0}\right)\left(I_{N_{\text {mod }}}-\mathcal{A} \bar{S}\left(z, \theta_{0}\right) \bar{G}\left(z, \theta_{0}\right) \bar{K}(z)\right)^{-1} \mathcal{A} \bar{m}_{l} \\
& \bar{S}\left(z, \theta_{0}\right)=\left(I_{N_{\text {mod }}}+\bar{G}\left(z, \theta_{0}\right) \bar{K}(z)\right)^{-1}
\end{aligned}
$$

For the sequel, it is important to note that $T_{\text {init }, l}(z)$ (see $(7)$ ) is the initial model of $T_{0, l}(z)$ that corresponds to the parameter $\theta_{\text {init }, l}$. Consequently, in (12), $T_{0, l}(z)-T_{\text {init }, l}(z)$ is the modeling error of this initial model $T_{\text {init,l }}(z)$.

With respect to the normal operations (3)-(4)-(5), $\bar{y}$ and $\bar{u}$ are thus perturbed during the identification experiment by $R^{y}(z) r(t)$ and $R^{u}(z) r(t)$, respectively. Consequently, it makes sense to define the cost of the identification experiment as:

$$
\begin{aligned}
& J\left(\Phi_{r}, \theta_{0}\right)=\frac{1}{2 \pi} \int_{-\pi}^{\pi}\left(\left(R^{y}\left(e^{j \omega}, \theta_{0}\right)\right)^{*} R^{y}\left(e^{j \omega}, \theta_{0}\right)+\eta\left(R^{u}\left(e^{j \omega}, \theta_{0}\right)\right)^{*} R^{u}\left(e^{j \omega}, \theta_{0}\right)\right) \Phi_{r}(\omega) d \omega \\
& J\left(\Phi_{r}, \theta_{0}\right)=\frac{1}{2 \pi} \int_{-\pi}^{\pi} \mathcal{R}^{*}\left(e^{j \omega}, \theta_{0}\right) \mathcal{R}\left(e^{j \omega}, \theta_{0}\right) \Phi_{r}(\omega) d \omega
\end{aligned}
$$

where $\eta$ is an user chosen weighting factor and where $\mathcal{R}\left(z, \theta_{0}\right)$ is the following vector of transfer functions of dimension $2 N_{\text {mod }}$ :

$$
\mathcal{R}\left(z, \theta_{0}\right)=\left(\begin{array}{c}
R^{y}\left(z, \theta_{0}\right) \\
\sqrt{\eta} R^{u}\left(z, \theta_{0}\right)
\end{array}\right)
$$

The cost $J\left(\Phi_{r}, \theta_{0}\right)$ can be rewritten as the sum of the individual costs $J_{i}\left(\Phi_{r}, \theta_{0}\right)\left(i=1, \ldots, N_{\text {mod }}\right)$ in each module:

$$
\begin{aligned}
J\left(\Phi_{r}, \theta_{0}\right)= & \sum_{i=1}^{N_{\text {mod }}} J_{i}\left(\Phi_{r}, \theta_{0}\right) \quad \text { with } \\
J_{i}\left(\Phi_{r}, \theta_{0}\right)= & \frac{1}{2 \pi} \int_{-\pi}^{\pi} \mathcal{R}_{i}^{*}\left(e^{j \omega}, \theta_{0}\right) \mathcal{R}_{i}\left(e^{j \omega}, \theta_{0}\right) \Phi_{r}(\omega) d \omega \\
& \mathcal{R}_{i}(z, \theta)=\left(\begin{array}{c}
R_{i}^{y}(z, \theta) \\
\sqrt{\eta} R_{i}^{u}(z, \theta)
\end{array}\right)
\end{aligned}
$$

where $R_{i}^{y}$ (resp. $R_{i}^{u}$ ) is the $i^{\text {th }}$ entry of $R_{y}$ (resp. $R^{u}$ ). Due to (13), we can also rewrite (18) as follows:

$$
J_{i}\left(\Phi_{r}, \theta_{0}\right)=\frac{1}{2 \pi} \int_{-\pi}^{\pi}\left(\left|G_{i}\left(e^{j \omega}, \theta_{0, i}\right)\right|^{2}+\eta\right)\left|R_{i}^{u}\left(e^{j \omega}, \theta_{0}\right)\right|^{2} \Phi_{r}(\omega) d \omega
$$


In the non-stealth setting, the cost $J\left(\Phi_{r}, \theta_{0}\right)$ of an identification experiment and the individual costs $J_{i}\left(\Phi_{r}, \theta_{0}\right)$ can be defined in a very similar way. However, the expressions for $R^{y}$ and $R^{u}$ that are used in (15) and (18) have a different expression:

$$
\begin{aligned}
& R^{u, N S}\left(z, \theta_{0}\right)=\bar{m}_{l} S_{0, l}(z)+\overline{\mathcal{N}}\left(z, \theta_{0}\right) T_{0, l}(z) \\
& R^{y, N S}\left(z, \theta_{0}\right)=\bar{G}\left(z, \theta_{0}\right) R^{u, N S}\left(z, \theta_{0}\right)
\end{aligned}
$$

By comparing (20) and (12), we observe that the modeling error $T_{0, l}(z)-T_{\text {init,l }}(z)$ is replaced by $T_{0, l}(z)$ in the expression of $R^{u, N S}$.

For both the stealth and non-stealth settings, using the definition of $\mathcal{P}_{l}$ (see Definition 1 ), the individual costs $J_{i}\left(\Phi_{r}, \theta_{0}\right)$ for $i \notin \mathcal{P}_{l}$ will be equal to zero. However, for $i \in \mathcal{P}_{l}$, these costs $J_{i}\left(\Phi_{r}, \theta_{0}\right)$ will be nonzero. Consequently, the excitation signal $r$ will not have an influence only on Node $l$ (where it is applied and where it is necessary for the identification of $\mathcal{S}_{l}$ ), but also on all nodes $i$ with $i \in \mathcal{P}_{l}$. For the network in Figure 1, if the excitation signal $r$ is applied in Node 5, besides $J_{5}\left(\Phi_{r}, \theta_{0}\right)$, the individual costs $J_{i}\left(\Phi_{r}, \theta_{0}\right)$ for $i=2,3,4$ and 6 will also be non-zero. This result is equivalent to the fact that $\mathcal{N}_{i}\left(z, \theta_{0}\right)$ (i.e. the $i^{\text {th }}$ entry of $\overline{\mathcal{N}}\left(z, \theta_{0}\right)$ ) is a nonzero transfer function for all $i \in \mathcal{P}_{l}$ and is equal to zero for all $i \neq l, i \notin \mathcal{P}_{l}$.

The role of the stealth compensation $x(t)$ is to reduce as much as possible this propagation of the influence of the excitation $r$ (applied in Node $l$ ) towards the nodes $i \in \mathcal{P}_{l}$. Before explaining this in more details, let us make the following assumptions on Node $l$ and the considered network:

Assumption 1 Consider the set $\mathcal{P}_{l}$ (see Definition 1) corresponding to the to-be-identified Node $l$. We assume that $\mathcal{P}_{l}$ is a non-empty set.

Assumption 2 Consider the set $\mathcal{P}_{l}$ (see Definition 1) corresponding to the to-be-identified Node $l$ and the vector of transfer functions $\mathcal{N}\left(z, \theta_{0}\right)$ (see (14)). We will assume that, for all $i \in \mathcal{P}_{l}$, the $i^{\text {th }}$ entry $\mathcal{N}_{i}\left(z, \theta_{0}\right)$ of $\mathcal{N}\left(z, \theta_{0}\right)$ is such that $\mathcal{N}_{i}\left(e^{j \omega}, \theta_{0}\right) \neq 0$ for (almost) all frequencies.

If $\mathcal{P}_{l}$ would be empty, there is of course no need for the stealth setting since the signal $r$ will not be propagated to other nodes. Assumption 2 will in fact always hold, except in pathological cases that we here want to formally exclude.

We can now explain the role of the stealth compensation in reducing the propagation of the influence of the excitation $r$ towards the nodes $i \in \mathcal{P}_{l}$. Let us first consider the ideal case i.e. when $T_{\text {init }, l}=T_{0, l}$. This choice does not change the situation in the non-stealth setting i.e. $J_{i}\left(\Phi_{r}, \theta_{0}\right)$ remains nonzero for all $i \in \mathcal{P}_{l}$ since $R_{i}^{u, N S}$ and $R_{i}^{y, N S}$ remains the same nonzero transfer functions for all these $i$ (they are not function of $T_{\text {init, },}$ ). However, in the stealth setting, for all $i \neq l$, the transfer functions $R_{i}^{u}$ and $R_{i}^{y}$ are identically zero when $T_{i n i t, l}=T_{0, l}$. Consequently, in this ideal case, the effect of the excitation $r(t)$ will only be felt in the to-be-identified module i.e. $J_{i}\left(\Phi_{r}, \theta_{0}\right)=0$ for all $i \neq l$.

In practice, $T_{i n i t, l}$ will of course always be different from $T_{0, l}$, but, as shown in the following proposition, the stealth configuration will remain beneficial if $T_{i n i t, l}$ satisfies a certain accuracy constraint that will hold in the vast majority of the cases.

Proposition 1 Consider that, following the procedure described in this section, an excitation signal $r(t)$ of spectrum $\Phi_{r}$ is applied to Node $l$ of a network like the one described in Section 2 and satisfying Assumptions 1 and 2. Let us for this spectrum $\Phi_{r}$ compute the individual costs $J_{i}\left(\Phi_{r}, \theta_{0}\right)$ $\left(i=1, \ldots, N_{\text {mod }}\right)$ in the stealth setting and in the non-stealth setting using the respective expression for these costs in the two settings (see Section 3.1). Suppose finally that the initial model $T_{\text {init }, l}(z)$ of $T_{0, l}(z)$ satisfies the following accuracy constraint at the frequencies $\omega$ where $\Phi_{r}(\omega) \neq 0$ :

$$
\frac{\left|T_{0, l}\left(e^{j \omega}\right)-T_{i n i t, l}\left(e^{j \omega}\right)\right|}{\left|T_{0, l}\left(e^{j \omega}\right)\right|}<1 .
$$


Then, for all $i \in \mathcal{P}_{l}$ (see Definition 1), the individual cost $J_{i}\left(\Phi_{r}, \theta_{0}\right)$ in the stealth configuration is strictly smaller than the one in the non-stealth configuration. Recall also that, for the nodes $i$ with $i \neq l$ and $i \notin \mathcal{P}_{l}, J_{i}\left(\Phi_{r}, \theta_{0}\right)=0$ in both configurations.

Proof. See Appendix A.

The property (22) is equivalent to say that, at $\omega, T_{\text {init,l }}$ is a better model of $T_{0, l}$ than the zero transfer function (i.e. $\left|T_{0, l}\left(e^{j \omega}\right)-T_{i n i t, l}\left(e^{j \omega}\right)\right|<\left|T_{0, l}\left(e^{j \omega}\right)-0\right|$ ). As already mentioned, this property will generally be met in practice. The advantage of the stealth configuration will be further discussed in the next section.

\subsection{Identification of one given module}

In Section 2, we assumed that we have obtained an initial estimate $\theta_{\text {init }}=\left(\theta_{\text {init }, 1}^{T}, \ldots, \theta_{\text {init }, N_{\text {mod }}}^{T}\right)^{T}$ of the true parameter vector $\theta_{0}=\left(\theta_{0,1}^{T}, \ldots, \theta_{0, N_{\text {mod }}}^{T}\right)^{T}$. As already mentioned at the end of Section 2, we will suppose that we want to increase the accuracy of the estimate ${ }^{3} \theta_{\text {init }, l}$ of the true parameter vector $\theta_{0, l}$ corresponding to Node $l$. The accuracy of $\theta_{\text {init }, l}$ can be measured with $P_{\text {init }, l}^{-1}$ where $P_{\text {init }, l}$ is the covariance matrix of $\theta_{\text {init }, l}$. The accuracy of $\theta_{\text {init, } l}$ can be improved by combining it with a new estimate of $\theta_{0, l}$ obtained using a data set $\left\{y_{l}(t), u_{l}(t) \mid t=1, \ldots, N\right\}$ collected as shown in Figure 3 in the stealth or the non-stealth setting.

We consider for this purpose a full-order model structure $\mathcal{M}=\left\{G_{l}\left(z, \theta_{l}\right), H_{l}\left(z, \theta_{l}\right) \mid \theta_{l} \in \mathbf{R}^{n_{l}}\right\}$ for $\mathcal{S}_{l}$. We suppose that $\mathcal{M}$ is globally identifiable at $\theta_{0, l}$ i.e., $\theta_{l}=\theta_{0, l}$ is the only parameter vector for which $G_{l}\left(z, \theta_{l}\right)$ and $H_{l}\left(z, \theta_{l}\right)$ corresponds to $\mathcal{S}_{l}$. We will also suppose that the excitation signal $r(t)$ (see Figure 3) and the white noise vector $\bar{e}$ (see (3)) are uncorrelated and that re $f_{\text {ext }}(t$ ) is a stationary signal uncorrelated with $r(t)$ and $\bar{e}(t)$. Then, using the data set $\left\{y_{l}(t), u_{l}(t) \mid t=\right.$ $1, \ldots, N\}$, an estimate $\hat{\theta}_{N, l}$ of $\theta_{0, l}$ can be obtained via prediction error identification [23]:

$$
\begin{aligned}
\hat{\theta}_{N, l} & =\arg \min _{\theta_{l}} \frac{1}{N} \sum_{t=1}^{N} \epsilon^{2}\left(t, \theta_{l}\right) \quad \text { with: } \\
\epsilon\left(t, \theta_{l}\right) & =H_{l}^{-1}\left(z, \theta_{l}\right)\left(y_{l}(t)-G_{l}\left(z, \theta_{l}\right) u_{l}(t)\right)
\end{aligned}
$$

Using a similar reasoning ${ }^{4}$ as in [2], we will show that $\hat{\theta}_{N, l}$ is a consistent estimate of $\theta_{0, l}$ i.e., $\theta_{0, l}$ is the unique minimum of the asymptotic criterion $\bar{E} \epsilon^{2}\left(t, \theta_{l}\right)=\lim _{N \rightarrow \infty} \frac{1}{N} \sum_{t=1}^{N} E \epsilon^{2}\left(t, \theta_{l}\right.$ ) (with $E$ the expectation operator). Let us for this purpose make a similar assumption as Assumption 2 to exclude pathological cases for the matrix $S^{u}$ in (11).

Assumption 3 Consider the set $\mathcal{L}_{l}$ (see Definition 1) corresponding to the to-be-identified Node $l$ and the matrix of transfer functions $S^{u}\left(z, \theta_{0}\right)$ (see (11)). We will assume that, for all $i \in \mathcal{L}_{l}$, the $(l, i)$ entry $S_{l i}^{u}\left(z, \theta_{0}\right)$ of $S^{u}\left(z, \theta_{0}\right)$ is such that $S_{l i}^{u}\left(e^{j \omega}, \theta_{0}\right) \neq 0$ for (almost) all frequencies $\omega$.

Proposition 2 Consider the network setup described in Section 2 and the identification procedure described above yielding the estimate $\hat{\theta}_{N, l}$ of $\theta_{0, l}$ using data collected as shown in Figure 3 (using the stealth or the non-stealth settings). Consider also the set $\mathcal{L}_{l}$ corresponding to Node $l$ (see

\footnotetext{
${ }^{3}$ This estimate $\theta_{i n i t, l}$ is also the one with which the transfer function $T_{i n i t, l}$ used for the stealth compensation is constructed.

${ }^{4}$ The main differences of Proposition 2 in this paper with respect to Theorem 1 in [2] are that we here consider the influence of $r e f_{\text {ext }}$ and that we also derive the consistency for the stealth setting.
} 
Definition 1) and suppose that Assumption 3 holds. Then, the estimate $\hat{\theta}_{N, l}$ is a consistent estimate of $\theta_{0, l}$ if the product $K_{l}(z) G_{l}\left(z, \theta_{0, l}\right)$ contains (at least) one delay and if one of the following two conditions is satisfied

- the set $\mathcal{L}_{l}$ is non-empty. In this case, $\hat{\theta}_{N, l}$ is consistent even if the external excitation $r(t)$ and the external reference signal re $f_{\text {ext }}$ are equal to zero.

- The order of excitation of $r$ (i.e. the number of frequencies at which its spectrum $\Phi_{r}(\omega)$ is nonzero in $]-\pi \pi]$ ) and the order of excitation of the external reference signal re $f_{\text {ext }}$ are sufficient for (23) to be consistent when the data set $\left\{y_{l}(t), u_{l}(t) \mid t=1, \ldots, N\right\}$ is collected in the following simple closed-loop system:

$$
\begin{array}{r}
y_{l}(t)=G_{l}\left(z, \theta_{0, l}\right) u_{l}(t)+H_{l}\left(z, \theta_{0, l}\right) e_{l}(t) \\
u_{l}(t)=r(t)+K_{l}(z)\left(r e f_{\text {ext }}(t)-y_{l}(t)\right)
\end{array}
$$

Proof. See Appendix B.1.

Let us interpret this proposition for the example of the network of Figure 1. In this network, $\mathcal{L}_{l}$ is non-empty for all nodes $l \neq 1$. For all these nodes, as shown in the proof of Proposition 2 , the perturbations $v_{i}(t)=H_{i}\left(z, \theta_{i, 0}\right) e_{i}(t)$ for $i \in \mathcal{L}_{l}$ will be sufficient to yield informative data $\left\{y_{l}(t), u_{l}(t) \mid t=1, \ldots, N\right\}$ and thus a consistent $\hat{\theta}_{N, l}$ (even if $r(t)=r e f_{\text {ext }}(t)=0$ ). It is however to be noted that, unless we have the luxury of performing an arbitrary long experiment, a nonzero excitation $r(t)$ will generally be required to obtain the desired accuracy for $\hat{\theta}_{N, l}$ (see later). As far as Node 1 is concerned, we see that this node can indeed be described as in (24)-(25). Consequently, the consistency of $\hat{\theta}_{N, l}$ can be checked using the data informativity result for direct closed loop identification (see e.g. [11]). In this paper, the consistency of $\hat{\theta}_{N, l}$ can be checked by verifying a condition on the excitation order of the excitation signal, which uniquely depends on the order of the controller $K_{l}(z)$ and on the respective parametrization and the orders of $G_{l}\left(z, \theta_{l}\right)$ and $H_{l}\left(z, \theta_{l}\right)$. Note that, since $r e f_{\text {ext }}$ is assumed quasi-stationary and independent of $r$, the orders of excitation of $r$ and $r e f_{\text {ext }}$ just add up.

We will from now on suppose that the estimate $\hat{\theta}_{N, l}$ is consistent. As shown in Appendix B.2, the estimate $\hat{\theta}_{N, l}$ is then also (asymptotically) normally distributed around $\theta_{0, l}$ with a covariance matrix $P_{\theta_{l}}$ that can be estimated from the data and whose inverse has the following expression:

$$
\begin{aligned}
& P_{\theta_{l}}^{-1}\left(\Phi_{r}, \theta_{0}\right)=M_{\bar{e}}\left(\theta_{0}\right)+ \\
& \frac{N}{2 \pi \sigma_{l}^{2}} \int_{-\pi}^{\pi} F_{l}\left(e^{j \omega}, \theta_{0, l}\right) F_{l}^{*}\left(e^{j \omega}, \theta_{0, l}\right)\left(\left|R_{l}^{u}\left(e^{j \omega}, \theta_{0}\right)\right|^{2} \Phi_{r}(\omega)+\left|R_{\text {ext }, l}^{u}\left(e^{j \omega}, \theta_{0}\right)\right|^{2} \Phi_{r e f_{\text {ext }}}(\omega)\right) d \omega
\end{aligned}
$$

with $R_{l}^{u}$ and $R_{e x t, l}^{u}$ the $l^{\text {th }}$ entry of $R^{u}$ and of $R_{e x t}^{u}$, respectively, and with $\Phi_{r e f_{e x t}}$ the power spectrum of refext, $F_{l}\left(z, \theta_{l}\right)=H_{l}^{-1}\left(z, \theta_{l}\right) \frac{\partial G_{l}\left(z, \theta_{l}\right)}{\partial \theta_{l}}$ and $M_{\bar{e}}\left(\theta_{0}\right)$ the contribution of $\bar{e}$ to the accuracy of the estimate (see Appendix B.2 for more details). We observe that $P_{\theta_{l}}^{-1}\left(\Phi_{r}, \theta_{0}\right)$ is an affine function of the power spectrum $\Phi_{r}$ of the excitation signal $r$ and of the power spectrum $\Phi_{r e f_{e x t}}$ of the external reference $r e f_{\text {ext }}$ (and a more complex function of $\theta_{0}$ ). Equation (26) pertains to the stealth configuration. In the non-stealth configuration, we can use the same expression for $P_{\theta_{l}}\left(\Phi_{r}, \theta_{0}\right)$, but we have to replace $R_{l}^{u}$ by $R_{l}^{u, N S}$ (see $(20)$ ).

We can combine the information on $\theta_{0, l}$ contained in the estimates $\hat{\theta}_{N, l}$ and $\theta_{\text {init }, l}$ using the following estimator $\hat{\theta}_{\text {final }, l}=\left(P_{\theta_{l}}^{-1}+P_{\text {init, }, l}^{-1}\right)^{-1}\left(P_{\theta_{l}}^{-1} \hat{\theta}_{N, l}+P_{i n i t, l}^{-1} \theta_{\text {init }, l}\right)$ whose covariance matrix is given by $\left(P_{\theta_{l}}^{-1}+P_{i n i t, l}^{-1}\right)^{-1}[22$, page 464$]$. 
The accuracy of the estimate $\hat{\theta}_{f i n a l, l}$ can thus be measured with $P_{\theta_{l}}^{-1}\left(\Phi_{r}, \theta_{0}\right)+P_{\text {init }, l}^{-1}$. In this paper, we will suppose that this accuracy will be deemed satisfactory if the following accuracy constraint $P_{\theta_{l}}^{-1}\left(\Phi_{r}, \theta_{0}\right)+P_{i n i t, l}^{-1}>R_{a d m}$ is satisfied. The matrix $R_{a d m}$ is a given strictly positive-definite and symmetric matrix that reflects the desired accuracy $[25,13]$.

\section{Optimal experiment design problem}

As mentioned in the introduction, we will design the spectrum $\Phi_{r}$ of the excitation signal $r$ of the identification experiment described in the previous section in such a way that the accuracy constraint $P_{\theta_{l}}^{-1}\left(\Phi_{r}, \theta_{0}\right)+P_{\text {init }, l}^{-1}>R_{a d m}$ is satisfied with the smallest cost $J\left(\Phi_{r}, \theta_{0}\right)$ (see (15)). This optimization problem can thus be formulated as follows:

$$
\begin{gathered}
\min _{\Phi_{r}} J\left(\Phi_{r}, \theta_{0}\right) \\
P_{\theta_{l}}^{-1}\left(\Phi_{r}, \theta_{0}\right)+P_{\text {init }, l}^{-1} \geq R_{a d m}
\end{gathered}
$$

This optimization problem can be considered both in the stealth and in the non-stealth setting by using the respective expressions for $J\left(\Phi_{r}, \theta_{0}\right)$ and for $P_{\theta_{l}}^{-1}\left(\Phi_{r}, \theta_{0}\right)$ in both cases. Before discussing how this optimization problem can be solved in practice, let us formulate the following result that illustrates the advantage of the stealth configuration.

Proposition 3 Consider an identification experiment in Node $l$ of a network satisfying Assumptions 1 and 2. Consider, for this identification experiment, the optimal experiment design problem (27)-(28) in the stealth and in the non-stealth setting (i.e. using the respective expressions for $J\left(\Phi_{r}, \theta_{0}\right)$ and for $P_{\theta_{l}}^{-1}\left(\Phi_{r}, \theta_{0}\right)$ in both cases) and let us denote by $\Phi_{r, o p t}^{S}$ and $\Phi_{r, o p t}^{N S}$ the optimal spectra obtained in these two settings. Then, we have that the optimal cost $J\left(\Phi_{r, o p t}^{S}, \theta_{0}\right)$ in the stealth setting is strictly smaller than the cost $J\left(\Phi_{r, o p t}^{N S}, \theta_{0}\right)$ in the non-stealth setting if the model $T_{\text {init }, l}$ of $T_{0, l}$ used in the stealth compensation (7) has the following property for all $\omega$ where $\Phi_{r, o p t}^{N S}(\omega) \neq 0$ :

$$
\frac{\left|T_{0, l}\left(e^{j \omega}\right)-T_{i n i t, l}\left(e^{j \omega}\right)\right|}{\left|T_{0, l}\left(e^{j \omega}\right)\right|}<\frac{\left|R_{l}^{u}\left(e^{j \omega}, \theta_{0}\right)\right|}{\left|R_{l}^{u, N S}\left(e^{j \omega}, \theta_{0}\right)\right|}
$$

where $R_{l}^{u}$ (resp. $\left.R_{l}^{u, N S}\right)$ is the $l^{\text {th }}$ entry of $R^{u}$ (resp. $R^{u, N S}$ ) defined in (12) (resp. (20)).

Proof. See Appendix C.

As shown in Proposition 3, we thus see that the stealth configuration, which can be very easily implemented in a multi-agent network, will be, in many cases, advantageous ${ }^{5}$ to obtain the required accuracy for the model of $\mathcal{S}_{l}$ with the smallest possible identification cost.

Let us now turn to the problem of solving the optimal experiment design problem (27)-(28). We observe that, like many optimal experiment design problems, this optimization problem is dependent on the unknown vector $\theta_{0}=\left(\theta_{0,1}^{T}, \ldots, \theta_{0, N_{m o d}}^{T}\right)^{T}$. Since $\theta_{0}$ is unknown, the optimization problem (27)-(28) cannot be tackled as such. A commonly used approach to circumvent this problem is to replace $\theta_{0}$ by an initial estimate. If we use the initial estimate $\theta_{\text {init }}$ for this purpose

\footnotetext{
${ }^{5}$ The condition (29) on $T_{\text {init }, l}$ is more complex than (22). However, (29) will be respected if $T_{\text {init }, l}$ is not a too poor estimate of $T_{0, l}$.
} 
(see Section 2), this would yield an optimization problem consisting in minimizing $J\left(\Phi_{r}, \theta_{\text {init }}\right)$ under the constraint $P_{\theta_{l}}^{-1}\left(\Phi_{r}, \theta_{\text {init }}\right)+P_{\text {init, } l}^{-1} \geq R_{a d m}$. In the stealth configuration, this approach will have a major disadvantage since $T_{\text {init }, l}$ in (7) is also computed based on $\theta_{\text {init }}$. The latter has as consequence that $J\left(\Phi_{r}, \theta_{\text {init }}\right)=J_{l}\left(\Phi_{r}, \theta_{\text {init }}\right)$ (because $J_{i}\left(\Phi_{r}, \theta_{\text {init }}\right)=0$ for all $i \neq l$ ). In other words, the propagation of the signal $r(t)$ towards the nodes $i$ with $i \in \mathcal{P}_{l}$ will not be taken into account in the optimal experiment design problem if we replace $\theta_{0}$ by $\theta_{\text {init }}$. We will therefore instead consider the following formulation where the cost constraint has been robustified using the initial uncertainty region $U_{\text {init }}$ (see (6)):

$$
\begin{gathered}
\min _{\Phi_{r}, \gamma} \gamma \\
J\left(\Phi_{r}, \theta\right) \leq \gamma \quad \forall \theta \in U_{\text {init }} \\
P_{\theta_{l}}^{-1}\left(\Phi_{r}, \theta_{\text {init }}\right)+P_{\text {init }, l}^{-1} \geq R_{a d m}
\end{gathered}
$$

This optimization problem can be considered both in the stealth and in the non-stealth settings by using the respective expressions for $J\left(\Phi_{r}, \theta\right)$ and for $P_{\theta_{l}}^{-1}\left(\Phi_{r}, \theta_{\text {init }}\right)$ in both cases.

As opposed to the case where $\theta_{0}$ is replaced by $\theta_{\text {init }}$, the above formulation will also take into account the propagation of the excitation signal in the stealth setting. In this setting, we will also observe a robustification of the stealth compensation. Indeed, the robustified formulation will favour spectra $\Phi_{r}$ yielding, for all $\theta \in U_{\text {init }}$, small perturbations $R_{i}^{u}(z, \theta) r(t)$ and $R_{i}^{y}(z, \theta) r(t)$ for $i=l$ and for $i \in \mathcal{P}_{l}$ (see (11)-(10)). For nodes $i \in \mathcal{P}_{l}$, this e.g. means that the power of the following signal has to be made small for all $\theta \in U_{\text {init }}$ :

$$
R_{i}^{u}(z, \theta) r(t)=\overline{\mathcal{N}}_{i}(z, \theta)\left(\frac{G_{l}(z, \theta)}{1+K_{l}(z) G_{l}(z, \theta)}-T_{i n i t, l}(z)\right) r(t)
$$

Consequently, the robustified optimal experiment design problem will generally and among other considerations favour spectra $\Phi_{r}(\omega)$ with more contributions in the frequency ranges where the stealth compensation will be more effective due to a small uncertainty of $T_{\text {init,l }}(z)$ (thereby robustifying the stealth configuration).

In both the stealth and non-stealth settings, the robustified optimal experiment design problem (30)-(32), has also the following properties. If we denote by $\Phi_{r, o p t}$ and $\gamma_{\text {opt }}$ the solution of this optimization problem, we have that $\gamma_{o p t}=\sup _{\theta \in U_{\text {init }}} J\left(\Phi_{r, o p t}, \theta\right)$. Since we assume that $\theta_{0} \in U_{\text {init }}$, this robustified formulation ensures that the a-priori unknown cost $J\left(\Phi_{r, o p t}, \theta_{0}\right)$ is guaranteed to be smaller than $\gamma_{\text {opt }}$. This would have not been the case if, instead of the robustified constraint (31), we would have used the non-robustified constraint $J\left(\Phi_{r}, \theta_{\text {init }}\right) \leq \gamma$.

Since the optimization problem (30)-(32) can be considered for the stealth and the non-stealth configurations, the solution of the optimization problem in both settings can be compared to verify whether the stealth configuration indeed yields a smaller cost. Note that this will generally be the case since, as explained above, the robust formulation will imply a robustification of the stealth compensation. However, in the case of (30)-(32), we do not have a condition such as (29) to guarantee this property.

\section{Tackling the robust cost constraint in a convex way}

Since (32) is affine in the decision variable $\Phi_{r}$, the optimization problem (30)-(32) will be convex if (31) can be transformed into a constraint linear in the decision variables $\Phi_{r}$ and $\gamma$. However, as very often in robustness analysis theory, we cannot find a tractable linear constraint that is equivalent to (31), but we will show in this section that we can find one that implies (31). Consequently, 
if we solve the optimization problem with this alternative constraint, its solution $\gamma_{\text {opt }}$ will be an upper bound for $\sup _{\theta \in U_{i n i t}} J\left(\Phi_{r, o p t}, \theta\right)$. This entails a certain conservatism, but this $\gamma_{o p t}$ remains an upper bound for the (unknown) cost $J\left(\Phi_{r, o p t}, \theta_{0}\right)$ of an identification experiment with spectrum $\Phi_{r, o p t}$.

In order to derive a tractable constraint implying (31), we have to take into account that we are in the network context and thus that the vector $\theta$ can be of high dimension. Consequently, instead of working directly on the uncertain vector $\theta$ as in [3], it is preferable to consider the so-called hierarchical approach. The hierarchical approach has indeed been introduced in [28] to analyze the robustness of large-scale (interconnected) systems. Consequently, our objective here will be to determine a tractable constraint implying (31) and that can be used in the context of the hierarchical approach.

To derive such a tractable linear constraint implying (31), we will assume that the to-bedesigned excitation signal $r$ is a multisine i.e., $r(t)=\sum_{m=1}^{L} A_{m} \sin \left(\omega_{m} t\right)$ where the frequencies $\omega_{m}$ $(m=1, \ldots, L)$ are fixed by the user (as e.g. a fine grid of the frequency range $[0 \pi]$ ) and where the amplitudes $A_{m}(m=1, \ldots, L)$ will be optimally determined. Such parametrization of the excitation signal is classically used in optimal experiment design and corresponds to the following spectrum:

$$
\Phi_{r}(\omega)=\pi \sum_{m=1}^{L} c_{m}\left(\delta\left(\omega-\omega_{m}\right)+\delta\left(\omega+\omega_{m}\right)\right) \geq 0 \forall \omega
$$

where $c_{m}=\frac{A_{m}^{2}}{2}(m=1, \ldots, L)$ will be the decision variables of the optimization problem. The positivity of $\Phi_{r}(\omega)$ for all $\omega$ can be imposed by the constraints $c_{m} \geq 0(m=1, \ldots, L)$ on these decision variables.

Using (33) and (15), the robust cost constraint (31) can be rewritten as follows:

$$
\sum_{m=1}^{L} c_{m}\left(\mathcal{R}^{*}\left(e^{j \omega_{m}}, \theta\right) \mathcal{R}\left(e^{j \omega_{m}}, \theta\right)\right) \leq \gamma \quad \forall \theta \in U_{\text {init }}
$$

In the next section, we will show that the hierarchical approach can be used to deduce, for each $\omega$, an accurate upper bound $\alpha(\omega)$ for

$$
\mathcal{J}_{w c}(\omega)=\sup _{\theta \in U_{\text {init }}}\left(\mathcal{R}^{*}\left(e^{j \omega}, \theta\right) \mathcal{R}\left(e^{j \omega}, \theta\right)\right)
$$

i.e. $J_{w c}(\omega) \leq \alpha(\omega)$. This computable upper bound $\alpha(\omega)$ for $J_{w c}(\omega)$ is important since it is a necessary ingredient to derive a tractable linear constraint implying the robust cost constraint (34) as shown in the following proposition.

Proposition 4 Consider the robust cost constraint (34) corresponding to a spectrum of the type (33). Then, the constraint (34) holds for a given $\gamma$ if the following inequality linear in the decision variables $c_{m}(m=1, \ldots, L)$ holds:

$$
\sum_{m=1}^{L} c_{m} \alpha\left(\omega_{m}\right) \leq \gamma
$$

where $\alpha(\omega)(m=1, \ldots, L)$ is an upper bound for $J_{w c}(\omega)$ (see(35))

Proof. For any frequency $\omega_{m}$, we have that: 


$$
\left(\mathcal{R}^{*}\left(e^{j \omega_{m}}, \theta\right) \mathcal{R}\left(e^{j \omega_{m}}, \theta\right)\right) \leq \alpha\left(\omega_{m}\right) \quad \forall \theta \in U_{\text {init }}
$$

The latter relation confirms that (34) holds if (36) holds.

Let us observe that (36) is an inequality constraint linear in the coefficients $c_{m}(m=1, \ldots, L)$. Moreover, since $\Phi_{r}$ is affine in $c_{m}(m=1, \ldots, L)$, the accuracy constraint (32) is also linear in these coefficients. Consequently, the following Linear Matrix Inequality (LMI) optimization problem is a convex formulation for the original robust optimal experiment design problem (30)-(32).

LMI formulation. Consider the parametrization (33) for the to-be-designed spectrum $\Phi_{r}$. For this parametrization, the LMI optimization problem has as decision variables a scalar $\gamma>0$ and coefficients $c_{m} \geq 0(m=1, \ldots, L)$ and consists in determining the smallest value of $\gamma$ for which both the LMI constraint (32) and the constraint (36) hold for some $c_{m} \geq 0(m=1, \ldots, L)$.

Let us denote by $c_{m, o p t}(m=1, \ldots, L)$ and by $\gamma_{o p t}$ the solution of this LMI optimization problem and let us also denote by $\Phi_{r, \text { opt }}$ the spectrum corresponding to the coefficients $c_{m, o p t}$. Then, due to Proposition 4, we have that $\gamma_{\text {opt }}$ is an upper bound of $\sup _{\theta \in U_{\text {init }}} J\left(\Phi_{r, \text { opt }}, \theta\right)$ and thus of $J\left(\Phi_{r, o p t}, \theta_{0}\right)$. Moreover, by construction, the spectrum $\Phi_{r, o p t}$ is also the one yielding the smallest value of (the upper bound of $\sup _{\theta \in U_{\text {init }}} J\left(\Phi_{r, o p t}, \theta\right)$ for which the accuracy constraint (32) is met.

Remark. The solution $\gamma_{o p t}=\sum_{m=1}^{L} c_{m, o p t} \alpha\left(\omega_{m}\right)$ of the optimization problem gives an upper bound $J^{u b}$ for the total cost $J\left(\Phi_{r, o p t}, \theta_{0}\right)$. We can also compute an upper bound $J_{i}^{u b}$ for the individual $\operatorname{costs} J_{i}\left(\Phi_{r, \text { opt }}, \theta_{0}\right)$ defined in (18). This upper bound $J_{i}^{u b}\left(i=1, \ldots, N_{\text {mod }}\right)$ is given by:

$$
J_{i}^{u b}=\sum_{m=1}^{L} c_{m, o p t} \alpha_{i}\left(\omega_{m}\right)
$$

where, for any frequency $\omega, \alpha_{i}(\omega)\left(i=1, \ldots, N_{\text {mod }}\right)$ is an upper bound for

$$
\mathcal{J}_{w c, i}(\omega)=\sup _{\theta \in U_{\text {init }}}\left(\mathcal{R}_{i}^{*}\left(e^{j \omega}, \theta\right) \mathcal{R}_{i}\left(e^{j \omega}, \theta\right)\right)
$$

Such an upper bound can also be computed using the tools that will be presented in the next section.

Remark. With respect to our earlier contribution [24], the approach that we propose in this paper to robustify the cost constraint is not based on any approximation.

\section{Computation of $\alpha(\omega)$ using the hierarchical approach}

\subsection{LFT representation of $\mathcal{R}(z, \theta)$}

To be able to use the LMI formulation of the optimal experiment design problem (30)-(32) given in the previous section, it is necessary to determine a way to compute an (accurate) upper bound $\alpha(\omega)$ for $J_{w c}(\omega)$ at a given ${ }^{6}$ frequency $\omega$. As mentioned before, the hierarchical approach will be here used for this purpose. In order to use the hierarchical approach for the problem at stake (see

\footnotetext{
${ }^{6}$ We will indeed need to determine $\alpha(\omega)$ at the $L$ frequencies present in (33).
} 
e.g. [2]), we first need to rewrite $\bar{s}(t)=\mathcal{R}(z, \theta) r(t)$ in a Linear Fractional Transformation (LFT) representation having a special form i.e., we have to determine vectors of signals $\bar{p}$ and $\bar{q}$ such that:

$$
\bar{p}(t)=\bar{T}(z, \theta) \bar{q}(t) \quad \text { and } \quad\left(\begin{array}{c}
\bar{q}(t) \\
\bar{s}(t)
\end{array}\right)=M(z)\left(\begin{array}{c}
\bar{p}(t) \\
r(t)
\end{array}\right)
$$

where $M(z)$ is not a function of $\theta$ and where $\bar{T}(z, \theta)$ is a diagonal matrix of dimension $N_{\bmod } \times N_{\bmod }$ for which the $(i, i)$ entry is a closed-loop transfer function related to an isolated ${ }^{7}$ loop made up of the controller $K_{i}$ and a model $G_{i}\left(z, \theta_{i}\right)$ of $G_{i}\left(z, \theta_{i, 0}\right)$. In our case, this upper part will be chosen as:

$$
\bar{T}(z, \theta)=\operatorname{diag}\left(T_{1}\left(z, \theta_{1}\right), \ldots, T_{N_{\text {mod }}}\left(z, \theta_{N_{\text {mod }}}\right)\right)
$$

where $T_{i}\left(z, \theta_{i}\right)=G_{i}\left(z, \theta_{i}\right) /\left(1+K_{i}(z) G_{i}\left(z, \theta_{i}\right)\right)$. Note that, in the sequel, we will often use the shorthand notation $\mathcal{R}(z, \theta)=\mathcal{F}(M(z), \bar{T}(z, \theta))$ for the LFT (39).

We will determine this LFT both in the stealth setting and in the non-stealth setting. Let us start with the stealth setting and let us pose $\bar{e}=0$ and $r e f_{e x t}=0$ in (3)-(8)-(9). If we express these equations for an arbitrary $\theta$, we obtain

$$
\begin{aligned}
& \bar{y}(t)=\bar{G}(z, \theta) \bar{u}(t) \\
& \bar{u}(t)=\bar{m}_{l} r(t)+\bar{K}(z)\left(\bar{y}_{r e f}(t)-\bar{y}(t)\right) \\
& \bar{y}_{r e f}(t)=\mathcal{A}(\bar{y}(t)-\bar{m}_{l} \underbrace{T_{i n i t, l}(z) r(t)}_{=x(t)})
\end{aligned}
$$

Inserting (42) in (41) yields:

$$
\bar{y}(t)=\bar{T}(z, \theta)\left(\bar{K}(z) \bar{y}_{r e f}(t)+\bar{m}_{l} r(t)\right)
$$

with the definition of $\bar{T}(z, \theta)$ given in (40). Let us now define $\bar{p}(t):=\bar{y}(t)$ and $\bar{q}(t):=\bar{K} \bar{y}_{\text {ref }}(t)+$ $\bar{m}_{l} r(t)$ and let us notice that, using $(43), \bar{q}(t)$ is equal to:

$$
\bar{q}(t)=\bar{K}(z) \mathcal{A}(\underbrace{\bar{y}(t)}_{\bar{p}(t)}-\bar{m}_{l} T_{\text {init }, l}(z) r(t))+\bar{m}_{l} r(t)
$$

Using (10) and (11), let us also notice that $\bar{s}(t)=\left(\bar{s}_{y}^{T}(t), \bar{s}_{u}^{T}(t)\right)^{T}$ with $\bar{s}_{y}(t)=R^{y}(z, \theta) r(t)=$ $\bar{y}(t)=\bar{p}(t)$ and $\bar{s}_{u}(t)=\sqrt{\eta} R^{u}(z, \theta) r(t)=\sqrt{\eta} \bar{u}(t)$ (when $\bar{e}=0$ and refext $=0$ ). Inserting (43) in (42) and using the fact that $\bar{y}(t)=\bar{p}(t)$, we obtain:

$$
\bar{u}(t)=\bar{K}(z)\left(\mathcal{A}-I_{N_{\text {mod }}}\right) \bar{p}(t)+\left(\bar{m}_{l}-\bar{K}(z) \mathcal{A} \bar{m}_{l} T_{\text {init,ll}}(z)\right) r(t)
$$

This therefore yields the following expression for $M(z)$ :

$$
M(z)=\left(\begin{array}{c|c}
\bar{K}(z) \mathcal{A} & \bar{m}_{l}-\bar{K}(z) \mathcal{A} \bar{m}_{l} T_{\text {init }, l}(z) \\
\hline I_{N_{\text {mod }}} & 0 \\
\sqrt{\eta} \bar{K}(z)\left(\mathcal{A}-I_{N_{\text {mod }}}\right) & \sqrt{\eta}\left(\bar{m}_{l}-\bar{K}(z) \mathcal{A} \bar{m}_{l} T_{\text {init }, l}(z)\right)
\end{array}\right)
$$

\footnotetext{
${ }^{7}$ By isolated, we means that the considered loop is not connected to other loops via a network connection.
} 
Let us now consider the non-stealth configuration. In this configuration, $R^{y}$ and $R^{u}$ (see (12)(13)) are replaced by $R^{y, N S}$ and $R^{u, N S}$ (see (20)-(21)) in the above reasoning. By doing this, $\mathcal{R}(z, \theta)$ can also be written as an $\operatorname{LFT} \mathcal{F}(M(z), \bar{T}(z, \theta))$ with

$$
M(z)=\left(\begin{array}{c|c}
\bar{K}(z) \mathcal{A} & \bar{m}_{l} \\
\hline I_{N_{\text {mod }}} & 0 \\
\sqrt{\eta} \bar{K}(z)\left(\mathcal{A}-I_{N_{\text {mod }}}\right) & \sqrt{\eta} \bar{m}_{l}
\end{array}\right)
$$

\subsection{Hierarchical approach}

Based on the LFT representation $\mathcal{R}(z, \theta)=\mathcal{F}(M(z), \bar{T}(z, \theta))$ deduced in the previous subsection (for both the stealth and non-stealth settings), we have now all the elements to derive the hierarchical approach to determine an upper bound $\alpha(\omega)$ for $J_{w c}(\omega)$ (see (35)). This approach is an adaptation of the procedure proposed in Section 3 of our previous contribution [2]. The main difference with [2] is that $\mathcal{R}(z, \theta)$ is here a vector of transfer function while, in [2], $\mathcal{R}(z, \theta)$ was a scalar transfer function.

Let us first notice that, at each frequency $\omega$, the frequency response $\bar{T}\left(e^{j \omega}, \theta\right)$ of every transfer matrix $\bar{T}(z, \theta)$ with $\theta \in U_{\text {init }}$ lies in the following multiplicative uncertainty region [33]:

$$
\begin{gathered}
\mathcal{T}(\omega)=\left\{\bar{T}\left(e^{j \omega}\right) \mid \bar{T}\left(e^{j \omega}\right)=\left(1+\Delta\left(e^{j \omega}\right)\right) \bar{T}\left(e^{j \omega}, \theta_{\text {init }}\right) \text { with } \Delta\left(e^{j \omega}\right) \in \boldsymbol{\Delta}(\omega)\right\} \\
\boldsymbol{\Delta}(\omega)=\left\{\Delta\left(e^{j \omega}\right)=\operatorname{diag}\left(\Delta_{1}\left(e^{j \omega}\right), \ldots, \Delta_{N_{\text {mod }}}\left(e^{j \omega}\right)\right)|| \Delta_{i}\left(e^{j \omega}\right)-\tilde{c}_{i}(\omega) \mid<\rho_{i}(\omega) i=1, \ldots, N_{\text {mod }}\right\}
\end{gathered}
$$

where, for each $i=1, \ldots, N_{\text {mod }}, \tilde{c}_{i}(\omega)$ is a complex scalar and $\rho_{i}(\omega)$ is a real scalar determined as follows:

$$
\begin{gathered}
\min _{\rho_{i}(\omega), \tilde{c}_{i}(\omega)} \rho_{i}(\omega) \\
\text { s.t. }\left|\tilde{T}_{i}\left(e^{j \omega}, \theta_{i}\right)-\tilde{c}_{i}(\omega)\right|<\rho_{i}(\omega) \quad \forall \theta_{i} \in U_{\text {init }, i}
\end{gathered}
$$

with $\tilde{T}_{i}\left(e^{j \omega}, \theta_{i}\right)=\frac{T_{i}\left(e^{j \omega}, \theta_{i}\right)-T_{i}\left(e^{j \omega}, \theta_{i n i t, i}\right)}{T_{i}\left(e^{j \omega}, \theta_{i n i t, i}\right)}$ and

$$
U_{\text {init }, i}=\left\{\theta_{i} \mid \theta \in U_{\text {init }}\right\}=\left\{\theta_{i} \mid\left(\theta_{i}-\theta_{\text {init }, i}\right)^{T} P_{\text {init }, i}^{-1}\left(\theta-\theta_{\text {init }, i}\right) \leq \chi_{\beta}\right\}
$$

By virtue of $(48), \mathcal{T}(\omega)$ is in fact the smallest multiplicative uncertainty region that contains $T\left(e^{j \omega}, \theta\right)$ for all $\theta \in U_{\text {init }}$. As shown in Appendix D, the quantities $\tilde{c}_{i}(\omega)$ and $\rho_{i}(\omega)$ can be exactly computed for each $i$ and for each $\omega$. Consequently, $\mathcal{T}(\omega)$ can thus be entirely defined.

Using the $\operatorname{LFT} \mathcal{R}(z, \theta)=\mathcal{F}(M(z), \bar{T}(z, \theta))$, the quantity $J_{w c}(\omega)$ at a given $\omega$ can be rewritten as:

$$
\left.J_{w c}(\omega)=\sup _{\theta \in U_{\text {init }}}\left(\mathcal{F}^{*}\left(M\left(e^{j \omega}\right), \bar{T}\left(e^{j \omega}, \theta\right)\right) \mathcal{F}\left(M\left(e^{j \omega}\right), \bar{T}\left(e^{j \omega}, \theta\right)\right)\right)\right)
$$

Using the multiplicative uncertainty region $\mathcal{T}(\omega)$ defined above, we have the following property:

$$
J_{w c}(\omega) \leq \underbrace{\left.\sup _{\bar{T}\left(e^{j \omega}\right) \in \mathcal{T}(\omega)}\left(\mathcal{F}^{*}\left(M\left(e^{j \omega}\right), \bar{T}\left(e^{j \omega}\right)\right) \mathcal{F}\left(M\left(e^{j \omega}\right), \bar{T}\left(e^{j \omega}\right)\right)\right)\right)}_{=\tilde{\alpha}(\omega)}
$$

Using simple transformation, the LFT $\mathcal{F}\left(M\left(e^{j \omega}\right), \bar{T}\left(e^{j \omega}\right)\right)$ in $\bar{T}\left(e^{j \omega}\right)$ can be transformed in an LFT in $\Delta\left(e^{j \omega}\right)$ i.e., $\mathcal{F}\left(M\left(e^{j \omega}\right), \bar{T}\left(e^{j \omega}\right)\right)=\mathcal{F}\left(M_{\Delta}\left(e^{j \omega}\right), \Delta\left(e^{j \omega}\right)\right)$ with

$$
M_{\Delta}\left(e^{j \omega}\right)=\left(\begin{array}{cc}
0 & \bar{T}\left(e^{j \omega}, \theta_{\text {init }}\right) \\
I_{N_{\text {mod }}} & \bar{T}\left(e^{j \omega}, \theta_{\text {init }}\right)
\end{array}\right) \star M\left(e^{j \omega}\right)
$$


Using this new LFT description, $\tilde{\alpha}(\omega)$ in $(50)$ can be rewritten equivalently as:

$$
\tilde{\alpha}(\omega)=\sup _{\Delta\left(e^{j \omega}\right) \in \boldsymbol{\Delta}(\omega)}\left(\mathcal{F}^{*}\left(M_{\Delta}\left(e^{j \omega}\right), \Delta\left(e^{j \omega}\right)\right) \mathcal{F}\left(M_{\Delta}\left(e^{j \omega}\right), \Delta\left(e^{j \omega}\right)\right)\right)
$$

An accurate upper bound for this quantity $\tilde{\alpha}(\omega)$ can be determined using the LMI optimization problem given in the following proposition. This computable upper bound for $\tilde{\alpha}(\omega)$ is thus also an upper bound for $J_{w c}(\omega)$ and will therefore be chosen as the quantity $\alpha(\omega)$ that is used in the LMI formulation of Section 5 .

Proposition 5 Consider a given frequency $\omega$ and the quantities $\rho_{i}(\omega)$ and $\tilde{c}_{i}(\omega)\left(i=1, \ldots, N_{\text {mod }}\right)$ defining the set $\mathcal{T}(\omega)$ (see (46)). Define $R_{\omega}$ (resp. $C_{\omega}$ ) as a diagonal matrix of dimension $N_{\text {mod }}$ whose elements are $\rho_{i}^{2}(\omega)$ (resp. $\left.\tilde{c}_{i}(\omega)\right)\left(i=1 \ldots, N_{\text {mod }}\right)$. Then, an upper bound for $\tilde{\alpha}(\omega)$ (see (51)) is given by $\tilde{\gamma}_{\text {opt }}(\omega)$ where $\tilde{\gamma}_{\text {opt }}(\omega)$ is the solution of the following LMI optimization problem. This LMI optimization problem has as decision variables a real scalar $\tilde{\gamma}(\omega)>0$ and a strictly positive definite diagonal matrix $T_{\omega} \in \mathbf{R}^{N_{\text {mod }} \times N_{\text {mod }}}$.

$$
\begin{aligned}
& \min _{\tilde{\gamma}(\omega), T_{\omega}} \tilde{\gamma}(\omega) \\
& \text { s.t. }\left(\begin{array}{c}
M_{\Delta}\left(e^{j \omega}\right) \\
I
\end{array}\right)^{*} \mathcal{N}(\tilde{\gamma}(\omega))\left(\begin{array}{c}
M_{\Delta}\left(e^{j \omega}\right) \\
I
\end{array}\right)<0
\end{aligned}
$$

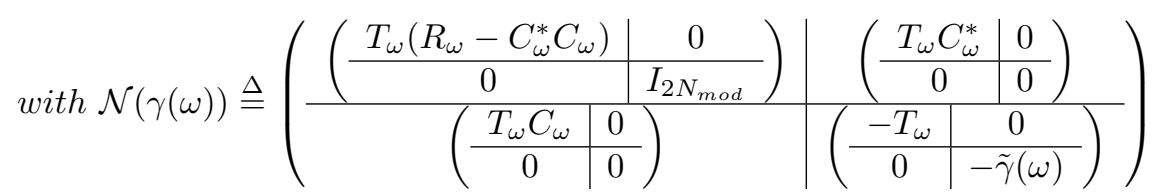

Proof. See Appendix E.

Remark. From a computational point-of-view, the hierarchical approach is advantageous for large networks. Indeed, due to the embedding in a multiplicative uncertainty, the complexity of the multiplier $T_{\omega}$ in the above proposition remains very limited (i.e., $N_{\text {mod }}$ elements in $T_{\omega}$ ). Moreover, the quantities $\rho_{i}(\omega)$ and $\tilde{c}_{i}(\omega)$ are computed locally in each node. In other words, the complexity of the network does not play a role in the computation of $\rho_{i}(\omega)$ and $\tilde{c}_{i}(\omega)$.

Remark. The quantity $\alpha_{i}(\omega)\left(i=1, \ldots, N_{m o d}\right)$ that can be used to determine the upper bound $J_{i}^{u b}$ on the individual cost $J_{i}$ (see (37)) can also be determined using the hierarchical approach presented above. For this purpose, we just have to replace, in the above reasoning, the LFT representation for $\mathcal{R}(z, \theta)$ by an LFT representation for $\mathcal{R}_{i}(z, \theta)$ (this LFT representation can be easily deduced from the one of $\mathcal{R}(z, \theta)$ ).

\section{Numerical illustrations}

\subsection{First numerical illustration}

In this first numerical illustration, we consider the network of Figure 1 made up of $N_{\text {mod }}=6$ homogenous nodes. In other words, the true systems $\mathcal{S}_{i}(i=1, \ldots, 6)$ are all identical and given by the following ARX system [21] with two resonance peaks: $y_{i}(t)=\left(z^{-3} B_{0}(z)\right) /\left(A_{0}(z)\right) u_{i}(t)+$ $(1) /\left(A_{0}(z)\right) e_{i}(t)$ with $B_{0}(z)=0.10276+0.18123 z^{-1}, A_{0}(z)=1-1.99185 z^{-1}+2.20265 z^{-2}-$ $1.84083 z^{-3}+0.89413 z^{-4}$. The variances of the white noises $e_{i}(t)$ are all equal to 0.5 . We further suppose that these true systems are all controlled by the same local controller $K(z)=\frac{K_{B}(z)}{K_{A}(z)}$ with 
$K_{B}(z)=0.03742-0.06719 z^{-1}+0.06995 z^{-2}-0.03814 z^{-3}-0.02546 z^{-4}+0.06323 z^{-5}-0.04707 z^{-6}+$ $0.03222 z^{-7}$ and $K_{A}(z)=1-3.348 z^{-1}+5.953 z^{-2}-7.163 z^{-3}+6.143 z^{-4}-3.705 z^{-5}+1.368 z^{-6}-$ $0.2482 z^{-7}$.

For simplicity, the initial estimate $\theta_{\text {init }}$ and its covariance matrix $P_{\text {init }}$ is determined from a single open-loop experiment on the ARX system (disconnected from the network) with a white input signal of variance 19 and of duration $N=1000$. This open-loop experiment yields the following identified polynomials $B_{i d}=0.09535 z^{-3}+0.1769 z^{-4}$ and $A_{i d}=1-1.989 z^{-1}+2.187 z^{-2}-1.822 z^{-3}+0.887 z^{-4}$ corresponding to the identified parameter vector $\theta_{\text {module,ol }}$ of dimension 6 with covariance matrix $P_{\text {module }, o l}$. Based on this open-loop experiment, the initial estimates $\theta_{\text {init }, i}$ of $\left.\theta_{0, i}(i=1, \ldots, 6)\right)$ can all be chosen equal to $\theta_{\text {module,ol }}$ i.e. $\theta_{\text {init }}=\left(\theta_{\text {module,ol }}^{T}, \theta_{\text {module,ol }}^{T}, \ldots, \theta_{\text {module,ol }}^{T}\right)^{T}$ and $P_{\text {init }}$ is chosen equal to $I_{N_{\text {mod }}} \otimes P_{\text {module,ol }}$. Using these quantities, we can define the uncertainty region $U_{\text {init }}$ corresponding to a confidence level $\beta$ of $95 \%\left(\chi_{\beta}=50.9985\right)$.

Our objective will be to design the spectrum $\Phi_{r}(\omega)$ of the excitation signal $r$ that has to be added to Node 5 (i.e. $l=5$ ) during an identification experiment of duration $N=1000$ to improve the accuracy of the model of $\mathcal{S}_{5}$ in such a way that the following accuracy constraint is satisfied $P_{\theta_{5}}^{-1}\left(\Phi_{r}, \theta_{0}\right)+P_{\text {init, } 5}^{-1}>R_{a d m}$ where $R_{a d m}$ is chosen as the inverse of the diagonal matrix $\operatorname{diag}\left((0.019)^{2},(0.022)^{2},(0.018)^{2},(0.009)^{2},(0.005)^{2},(0.009)^{2}\right)$. We will furthermore suppose that $r e f_{\text {ext }}(t)=0$ during the identification experiment.

To determine the spectrum $\Phi_{r}(\omega)$ satisfying this accuracy constraint with the smallest identification cost, we consider the optimization problem (30)-(32). We will consider this optimization problem both in the stealth setting and in the non-stealth setting. For both settings, we define the cost of the identification experiment as in (15) with $\eta=1$ and we parametrize $\Phi_{r}(\omega)$ as in (33) with $L=20$ frequencies distributed in the frequency range $\left[10^{-2}, \pi\right]$. In the stealth setting, the stealth compensation is implemented using (7) with the transfer function $T_{\text {init }, 5}$ that can be constructed based on $\theta_{\text {init }, 5}=\theta_{\text {module }, o l}$.

To solve the optimization problem (30)-(32) in both settings, we compute, for each setting, the corresponding quantity $\alpha(\omega)$ for each frequencies $\omega_{m}(m=1, \ldots, L)$ present in $\Phi_{r}(\omega)$ (see Section 6). The optimal spectrum $\Phi_{r, o p t}$ and the optimal value $\gamma_{o p t}$ can then be deduced ${ }^{8}$ using the LMI optimization problem given at the end of Section 5.

Let us first consider the stealth setting. In this case, the optimal spectrum $\Phi_{r, o p t}$ corresponds to a multisine for which all the amplitudes are negligible except at two frequencies $\omega=1.35$ and $\omega=1.65$ and $\gamma_{\text {opt }}$ is equal to 34.9466. This value is an upper bound $J^{u b}$ for $\sup _{\theta \in U_{\text {init }}} J\left(\Phi_{r, o p t}, \theta\right)$ as discussed in Section 4 and thus also an upper bound for the a-priori unknown cost $J\left(\Phi_{r, o p t}, \theta_{0}\right)$. As mentioned in the beginning of Section 3, our LMI approach entails a certain conservatism. Let us thus check the extent of this conservatism in this example. For this purpose, we compute a lower bound $J^{l b}$ for $\sup _{\theta \in U_{\text {init }}} J\left(\Phi_{r, \text { opt }}, \theta\right)$ by considering the maximal value of $J\left(\Phi_{r, \text { opt }}, \theta_{i}\right)$ over a set of 1000 grid points $\theta_{i} \in U_{\text {init }}$. This procedure yields $J^{l b}=33.92$. Consequently, the conservatism of our procedure remains limited in this example since there is only $3 \%$ of difference between $J^{l b}$ and $J^{u b}$. In this simulation example, we can also compute $J\left(\Phi_{r, o p t}, \theta_{0}\right)$ i.e. $J\left(\Phi_{r, o p t}, \theta_{0}\right)=31.39$. Consequently, $\gamma_{o p t}$ is indeed an upper bound for $J\left(\Phi_{r, o p t}, \theta_{0}\right)$. Note also that, as opposed to this, $J\left(\Phi_{r, \text { opt }}, \theta_{\text {init }}\right)=30.79$ would underestimate the actual cost of the experiment.

In order to have a better idea of how this cost is distributed in each of the six modules, let us consider the upper bound $J_{i}^{u b}$ (see $(37)$ ) of the individual costs $J_{i}\left(\Phi_{r, \text { opt }}, \theta_{0}\right)$ for $i=5$ and for all $i \in \mathcal{P}_{5}=\{2,3,4,6\}$. These upper bounds are given in Table 1 . We observe that $J_{5}^{u b}$ is almost equal to the total cost (for $\left.i \neq 5, J_{i}^{u b} \leq 3.510^{-4}\right)$.

Let us now compare these results with the ones obtained in the non-stealth setting. The optimal multisine in this setting is very similar to the one in the stealth setting and $\gamma_{o p t}=34.9557$. We

\footnotetext{
${ }^{8}$ Note that the whole procedure yielding $\Phi_{r, \text { opt }}$ and $\gamma_{o p t}$ takes 11 seconds using Matlab.
} 
observe that this value is (slightly) larger than in the stealth case where $\gamma_{\text {opt }}=34.9466$. Moreover, as shown in Table 1 , the costs $J_{i}^{u b}$ for $i \in \mathcal{P}_{5}$ are all at least 16 times larger than when the stealth configuration is implemented. This shows the advantage of the stealth configuration. The maximum of these individual costs for $i \neq 5$ (i.e. $J_{6}^{u b}=610^{-3}$ ) remains though small with respect to $J_{5}^{u b}=34.9470$. This is due to the large attenuation of this particular network. In other networks, this attenuation could be much smaller and would even more justify the use of the stealth configuration. This will be illustrated in the second numerical illustration.

Table 1: Upper bounds $J_{i}^{u b}$ for the individual costs $J_{i}(i=2, \ldots, 6)$ obtained using the optimal spectrum in the stealth and non-stealth settings (first illustration)

\begin{tabular}{|c||c|c|c|c|c|}
\hline & $J_{2}^{u b}$ & $J_{3}^{u b}$ & $J_{4}^{u b}$ & $J_{5}^{u b}$ & $J_{6}^{u b}$ \\
\hline STEALTH & $710^{-9}$ & $8.810^{-5}$ & $8.810^{-5}$ & 34.9461 & $3.510^{-4}$ \\
\hline NON-STEALTH & $1.210^{-7}$ & $1.510^{-3}$ & $1.510^{-3}$ & 34.9470 & $610^{-3}$ \\
\hline
\end{tabular}

\subsection{Second numerical illustration}

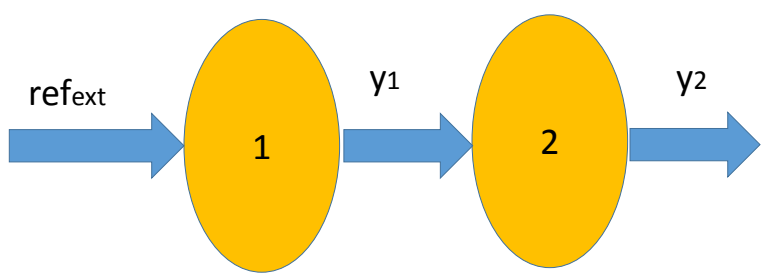

Figure 4: Graph representation of the network used in the second numerical illustration

In this second numerical illustration, we consider the network of Figure 4 made up of $N_{\text {mod }}=2$ nodes in cascade for which we wish to identify the first node. In this network, the matrices $\mathcal{A}$ and $\mathcal{B}$ in the interconnection (9) are given by

$$
\mathcal{A}=\left(\begin{array}{ll}
0 & 0 \\
1 & 0
\end{array}\right) \quad \mathcal{B}=\left(\begin{array}{l}
1 \\
0
\end{array}\right)
$$

The true system $\mathcal{S}_{1}$ is equal to the ARX system used in the previous section while the true system $\mathcal{S}_{2}$ is given by the following first-order ARX system: $y_{2}(t)=\left(3.6 z^{-1}\right) /\left(1-0.7 z^{-1}\right) u_{2}(t)+1 /(1-$ $\left.0.7 z^{-1}\right) e_{2}(t)$ (i.e. $\left.\theta_{0,2}=(-0.73 .6)^{T}\right)$. The controller $K_{1}$ is also the same as the one in the previous subsection while $K_{2}(z)=10$. The variance of the white noises $e_{1}(t)$ and $e_{2}(t)$ are here also both equal to 0.5. Note that the controller $K_{2}$ ensures a large closed-loop bandwidth. Consequently, in the non-stealth configuration, any perturbation due to $r(t)$ in $y_{r e f, 2}(t)=y_{1}(t)$ will have a strong influence on $u_{2}(t)$ and $y_{2}(t)$. A stealth configuration may thus have a large impact in this example.

For simplicity, the initial estimate $\theta_{\text {init }, 1}$ and its covariance matrix $P_{\text {init, } 1}$ will be chosen as in the previous section (i.e. the estimate obtained with an open-loop experiment with input variance of 19). The initial estimate $\theta_{\text {init }, 2}$ and its covariance matrix $P_{\text {init }, 2}$ are determined in a similar way 
(open-loop experiment with input variance of 19). This yields $\theta_{i n i t, 2}=(-0.693 .57)^{T}$ and a covariance matrix $P_{\text {init }, 2}$. Based on this, the initial estimate $\theta_{\text {init }}$ of $\theta_{0}$ is chosen as $\left(\theta_{\text {init, } 1}^{T}, \theta_{\text {init, }, 2}^{T}\right)^{T}$ and $P_{\text {init }}$ is chosen equal to $\operatorname{bdiag}\left(P_{\text {init }, 1}, P_{\text {init }, 2}\right)$. Using these quantities, we can define the uncertainty region $U_{\text {init }}$ corresponding to a confidence level $\beta$ of $95 \%\left(\chi_{\beta}=15.5\right)$.

Our objective will be to design the spectrum $\Phi_{r}(\omega)$ of the excitation signal $r$ that has to be added to Node $1(l=1)$ during an identification experiment of duration $N=1000$ to improve the accuracy of the model of $\mathcal{S}_{1}$ in such a way that the following accuracy constraint is satisfied $P_{\theta_{1}}^{-1}\left(\Phi_{r}, \theta_{0}\right)+P_{\text {init }, 1}^{-1}>R_{a d m}$ where $R_{a d m}$ is chosen as in the previous subsection. We will here also suppose that $r e f_{\text {ext }}(t)=0$ during the identification experiment.

To determine the spectrum $\Phi_{r}(\omega)$ satisfying this accuracy constraint with the smallest identification cost, we consider the optimization problem (30)-(32). We will consider this optimization problem here also both in the stealth setting and in the non-stealth setting. For both settings, we here also define the cost of the identification experiment as in (15) with $\eta=1$ and we parametrize $\Phi_{r}(\omega)$ as in (33) with the same $L=20$ frequencies as in the previous subsection. In the stealth setting, the stealth compensation is implemented using (7) with the transfer function $T_{\text {init, } 1}$ that can be constructed based on $\theta_{\text {init, } 1}$ ( $T_{\text {init }, 1}$ is here equal to the transfer function $T_{\text {init }, 5}$ in the previous subsection)

Let us compare the value $\gamma_{\text {opt }}$ obtained when solving (30)-(32) in the stealth and in the nonstealth settings. Like in the previous subsection, the optimal cost $\gamma_{o p t}$ in the stealth setting is smaller than in the non-stealth setting: the obtained $\gamma_{\text {opt }}$ are respectively 33.87 and 42.38 . Note that the advantage of the stealth configuration is here very clear since the stealth configuration allows to reduce the predicted cost by $25 \%$.

In order to have a better idea of how this cost is distributed in each of the two modules in both settings, we can consider Table 2 giving the upper bound $J_{i}^{u b}$ of the individual costs $J_{i}\left(\Phi_{r, o p t}, \theta_{0}\right)$ $(i=1,2)$. By comparing $J_{2}^{u b}=0.17$ (in the stealth setting) and $J_{2}^{u b}=8.61$ (in the non-stealth setting), we observe that the stealth configuration plays its role entirely by reducing, by a factor of 50 , the propagation of the excitation signal to Node 2. This strong reduction is also observed for the actual individual cost $J_{2}\left(\Phi_{r, o p t}, \theta_{0}\right)$ which is equal to 0.03 in the stealth setting and to 7.04 in the non-stealth setting.

Table 2: Upper bounds $J_{i}^{u b}$ for the individual costs $J_{i}(i=1,2)$ obtained using the optimal spectrum in the stealth and non-stealth settings in the second numerical illustration

\begin{tabular}{|c||c|c|}
\hline & $J_{1}^{u b}$ & $J_{2}^{u b}$ \\
\hline STEALTH & 33.70 & 0.17 \\
\hline NON-STEALTH & 33.77 & 8.61 \\
\hline
\end{tabular}

\subsection{Case of a larger $U_{\text {init }}$}

To show the effectiveness of the stealth configuration even in the case of a larger uncertainty $U_{\text {init }}$ (and thus a worse model $T_{\text {init,l }}$ ), we repeat the above comparisons when $\theta_{\text {init }}$ is identified with an input signal with a variance 10 times smaller than previously. In the first numerical illustration, this larger $U_{\text {init }}$ yields a value of $\gamma_{o p t}=131.66$ in the stealth setting and a value of $\gamma_{o p t}=131.71$ in the non-stealth one. In the second numerical illustration, we obtain $\gamma_{o p t}=119$ in the stealth setting and $\gamma_{o p t}=157.84$ in the non-stealth setting. Consequently, with this larger $U_{\text {init }}$ and this worse model $T_{\text {init, }, l}$, the stealth configuration also yields a smaller identification cost than the non-stealth configuration. Even though the obtained reduction is smaller due to the worse model $T_{\text {init }, l}$, the 
effectiveness of the stealth setting in reducing the propagation of the influence of the excitation signal remains confirmed when looking at Tables 3 and 4 giving, for this larger $U_{\text {init }}$ and this worse $T_{\text {init }, l}$, the upper bounds $J_{i}^{u b}$ of the individual costs $J_{i}\left(\Phi_{r, \text { opt }}, \theta_{0}\right)$ for the first and second numerical illustrations, respectively. In Table 3, we observe that $J_{i}^{u b}$ for $i \in \mathcal{P}_{5}$ remain 4.5 times smaller (instead of 16 times smaller in Table 1) when the stealth configuration is implemented. In Table 4, $J_{2}^{u b}$ remains 7 times smaller (instead of 50 times smaller in Table 2) when the stealth configuration is implemented.

Table 3: Upper bounds $J_{i}^{u b}$ for the individual costs $J_{i}(i=2, \ldots, 6)$ obtained using the optimal spectrum in the stealth and non-stealth settings for a larger $U_{\text {init }}$ (first numerical illustration)

\begin{tabular}{|c||c|c|c|c|c|}
\hline & $J_{2}^{u b}$ & $J_{3}^{u b}$ & $J_{4}^{u b}$ & $J_{5}^{u b}$ & $J_{6}^{u b}$ \\
\hline STEALTH & $310^{-7}$ & $2.110^{-3}$ & $2.110^{-3}$ & 131.649 & $8.310^{-3}$ \\
\hline NON-STEALTH & $1.310^{-6}$ & $9.410^{-3}$ & $9.510^{-3}$ & 131.656 & $3.810^{-2}$ \\
\hline
\end{tabular}

Table 4: Upper bounds $J_{i}^{u b}$ for the individual costs $J_{i}(i=1,2)$ obtained using the optimal spectrum in the stealth and non-stealth settings for a larger $U_{\text {init }}$ (second numerical illustration)

\begin{tabular}{|c||c|c|}
\hline & $J_{1}^{u b}$ & $J_{2}^{u b}$ \\
\hline STEALTH & 113.57 & 5.43 \\
\hline NON-STEALTH & 118.12 & 39.72 \\
\hline
\end{tabular}

It is to be noted that, for both numerical illustrations, the condition (29) is verified at all frequencies even with the worse model $T_{i n i t, l}$ considered in this section. Consequently, by virtue of Proposition 3, the result presented above could have been expected. However, note that this condition (29) cannot be verified in practice since it requires the knowledge of the unknown true system and that Proposition 3 only pertains to the ideal optimal experiment design problem (27)(28) and not to (30)-(32).

\section{Conclusions}

This paper extends the least costly identification experiment design framework to the case of the identification of one module in a network of locally controlled systems. The cost of the identification experiment (that is minimized under a certain accuracy constraint) is here defined as a function of the perturbations induced by the excitation signal on the input and output signals of each module. The propagation of the influence of the excitation signal is further reduced by an extension of the stealth identification paradigm. Note that the results of this paper can easily be extended to network situation where the cost of the experiment is not related to the perturbation induced by the excitation signal on the output of each module, but is more related to the perturbation induced by the excitation signal on the differences between the outputs of neighbouring modules. This situation can occur when the different agents in the network must remain as much as possible in a given formation configuration [29]. 


\section{References}

[1] X. Bombois, M. Gevers, G. Scorletti, and B.D.O. Anderson. Robustness analysis tools for an uncertainty set obtained by prediction error identification. Automatica, 37(10):1629-1636, 2001.

[2] X. Bombois, A. Korniienko, H. Hjalmarsson, and G. Scorletti. Optimal identification experiment design for the interconnection of locally controlled systems. Automatica, 89:169-179, 2018 .

[3] X. Bombois, F. Morelli, H. Hjalmarsson, L. Bako, and K. Colin. Robust optimal identification experiment design for multisine excitation. Submitted to Automatica, 2019. Available on the HAL repository under the reference hal-02363067.

[4] X. Bombois, G. Scorletti, M. Gevers, P.M.J. Van den Hof, and R. Hildebrand. Least costly identification experiment for control. Automatica, 42(10):1651-1662, 2006.

[5] A. Dankers, P. Van den Hof, X. Bombois, and P. Heuberger. Identification of dynamic models in complex networks with prediction error methods - predictor input selection. IEEE Transactions on Automatic Control, 61(4):937-952, 2016.

[6] N. Everitt, G. Bottegal, C. Rojas, and H. Hjalmarsson. On the variance analysis of identified linear MIMO models. In Proc. 54th IEEE Conference on Decision and Control, pages 14471452, Osaka, Japan, 2015.

[7] N. Everitt, H. Hjalmarsson, and C.R. Rojas. A geometric approach to variance analysis of cascaded systems. In Proceedings 52st IEEE Conference on Decision and Control, Florence, Italy, 2013.

[8] N. Everitt, H. Hjalmarsson, and C.R. Rojas. Variance results for parallel cascade serial systems. In The 19th IFAC World Congress, Cape Town, South Africa, 2014.

[9] J.A. Fax and R.H. Murray. Information flow and cooperative control of vehicle formations. IEEE Transactions on Automatic Control, 49(9):1465-1476, 2004.

[10] M. Forgione, X. Bombois, and P.M.J. Van den Hof. Data-driven model improvement for model-based control. Automatica, 52:118-124, 2015.

[11] M. Gevers, A. S. Bazanella, and L. Miskovic. Informative data: how to get just sufficiently rich? In Proc. 47th IEEE Conference on Decision and Control, pages pp. 1962-1967, Cancun, Mexico, 2008.

[12] M. Gevers and A.S. Bazanella. Identification in dynamic networks: identifiability and experiment design issues. In Proc. 54th IEEE Conference on Decision and Control, pages 4005-4010, Osaka, Japan, 2015.

[13] D. Ghosh, X. Bombois, J. Huillery, G. Scorletti, and G. Mercère. Optimal identification experiment design for lpv systems using the local approach. Automatica, 87:258-266, 2018.

[14] B. Gunes, A. Dankers, and P. Van den Hof. A variance reduction technique for identification in dynamic networks. In Proc. IFAC World Congress, pages 2842-2847, Cape Town, South Africa, 2014. 
[15] A. Haber and M. Verhaegen. Moving horizon estimation for large-scale interconnected systems. IEEE Transactions on Automatic Control, 58(11):2834-2847, 2013.

[16] P. Hägg and B. Wahlberg. On identification of parallel cascade serial systems. In Proc. IFAC World Congress, pages 9978-9983, Cape Town, South Africa, 2014.

[17] P. Hägg and B. Wahlberg. On optimal input design for networked systems. Automatica, 53:275 $-281,2015$.

[18] J. Hendrickx, M. Gevers, and A. Bazanella. Identifiability of dynamical networks with partial node measurements. to appear in IEEE Transactions on Automatic Control, June 2019.

[19] A. Korniienko, G. Scorletti, E. Colinet, and E. Blanco. Performance control for interconnection of identical systems: Application to pll network design. International Journal of Robust and Nonlinear Control, 2014.

[20] A. Kumar and S. Narasimhan. Robust plant friendly optimal input design. IFAC Proceedings Volumes, 46(32):553-558, 2013.

[21] ID Landau, Daniel Rey, Alireza Karimi, Alina Voda, and A Franco. A flexible transmission system as a benchmark for robust digital control. European Journal of Control, 1(2):77-96, 1995 .

[22] L. Ljung. System Identification: Theory for the User, 2nd Edition. Prentice-Hall, Englewood Cliffs, NJ, 1999.

[23] L Ljung. System Identification-Theory for the User 2nd edition PTR Prentice-Hall. Upper Saddle River, USA, 1999.

[24] F. Morelli, X. Bombois, H. Hjalmarsson, L. Bako, and K. Colin. Optimal experiment design for the identification of one module in the interconnection of locally controlled systems. In European Control Conference, pages 363-368, Naples, 2019.

[25] M. Potters. Experiment design for identification of structured linear systems. PhD thesis, Delft University of Technology, 2016.

[26] M. Potters, X. Bombois, M. Forgione, P. Modén, M. Lundh, H. Hjalmarsson, and P. Van den Hof. Optimal experiment design in closed loop with unknown, nonlinear and implicit controllers using stealth identification. In European Control Conference (ECC), pages 726-731, 2014.

[27] C. Rojas, J. Welsh, G. Goodwin, and A. Feuer. Robust optimal experiment design for system identification. Automatica, 43(6):993-1008, 2007.

[28] M. G. Safonov. Propagation of conic model uncertainty in hierarchical systems. IEEE Transactions on Circuits and Systems, pages 388-396, June 1983.

[29] P. Seiler, A. Pant, and K. Hedrick. Disturbance propagation in vehicle strings. IEEE Transactions on automatic control, 49(10):1835-1842, 2004.

[30] T. Vincent, C. Novara, K. Hsu, and K. Poolla. Input design for structured nonlinear system identification. Automatica, 46:990-998, 2010.

[31] B. Wahlberg, H. Hjalmarsson, and J. Mårtensson. Variance results for identification of cascade systems. Automatica, 45(6):1443-1448, 2009. 
[32] H. Weerts, A. Dankers, and P. Van den Hof. Identifiability in dynamic network identification. In Proc. IFAC Symposium on System Identification, pages 1409-1414, Beijing, China, 2015.

[33] K. Zhou and J. Doyle. Essentials of Robust Control. Prentice Hall, Upper Saddle River, New Jersey, 1998.

\section{A Proof of Proposition 1}

Let us consider the expression (19) for the individual costs $J_{i}\left(\Phi_{r}, \theta_{0}\right)\left(i=1, \ldots, N_{\text {mod }}\right)$ and introduce the following notations to distinguish them in the stealth (superscript $S$ ) and non-stealth case (superscript $N S$ ):

$$
\begin{gathered}
J_{i}^{S}\left(\Phi_{r}, \theta_{0}\right)=\frac{1}{2 \pi} \int_{-\pi}^{\pi}\left(\left|G_{i}\left(e^{j \omega}, \theta_{0, i}\right)\right|^{2}+\eta\right)\left|R_{i}^{u}\left(e^{j \omega}, \theta_{0}\right)\right|^{2} \Phi_{r}(\omega) d \omega \\
J_{i}^{N S}\left(\Phi_{r}, \theta_{0}\right)=\frac{1}{2 \pi} \int_{-\pi}^{\pi}\left(\left|G_{i}\left(e^{j \omega}, \theta_{0, i}\right)\right|^{2}+\eta\right)\left|R_{i}^{u, N S}\left(e^{j \omega}, \theta_{0}\right)\right|^{2} \Phi_{r}(\omega) d \omega
\end{gathered}
$$

where $R^{u}$ is given by (12) and $R^{u, N S}$ is given by (20). Let us now observe that, for $i \neq l$, we have that:

$$
\begin{gathered}
\left|R_{i}^{u}\left(e^{j \omega}, \theta_{0}\right)\right|^{2} \Phi_{r}(\omega)=\left|\bar{N}_{i}\left(e^{j \omega}, \theta_{0}\right)\right|^{2}\left|T_{0, l}\left(e^{j \omega}\right)-T_{i n i t, l}\left(e^{j \omega}\right)\right|^{2} \Phi_{r}(\omega) \\
\left|R_{i}^{u, N S}\left(e^{j \omega}, \theta_{0}\right)\right|^{2} \Phi_{r}(\omega)=\left|\bar{N}_{i}\left(e^{j \omega}, \theta_{0}\right)\right|^{2}\left|T_{0, l}\left(e^{j \omega}\right)\right|^{2} \Phi_{r}(\omega)
\end{gathered}
$$

Let us first consider the indexes $i \in \mathcal{P}_{l}$ and recall Assumption 2. In this case, if $T_{\text {init, } l \text { satisfies }}(22)$ for all frequencies $\omega$ where $\Phi_{r}(\omega) \neq 0$, we have for all these frequencies that:

$$
\left|R_{i}^{u, N S}\left(e^{j \omega}, \theta_{0}\right)\right|^{2} \Phi_{r}(\omega)>\left|R_{i}^{u}\left(e^{j \omega}, \theta_{0}\right)\right|^{2} \Phi_{r}(\omega)
$$

Consequently, for all $i \in \mathcal{P}_{l}$, we have that $J_{i}^{S}\left(\Phi_{r}, \theta_{0}\right)<J_{i}^{N S}\left(\Phi_{r}, \theta_{0}\right)$.

Finally, for the indexes $i \neq l, i \notin \mathcal{P}_{l}, J_{i}^{S}\left(\Phi_{r}, \theta_{0}\right)=J_{i}^{N S}\left(\Phi_{r}, \theta_{0}\right)=0$ since $\bar{N}_{i}\left(z, \theta_{0}\right)=0$ in this case.

\section{B Consistency and accuracy of (23)}

\section{B.1 Proof of Proposition 2}

Let us first consider the stealth setting. To show the consistency of (23), we have to show that $\theta_{0, l}$ is the unique minimum of the following asymptotic identification criterion:

$$
\theta_{l}^{*}=\arg \min _{\theta} \bar{E} \epsilon^{2}\left(t, \theta_{l}\right)
$$

The prediction error $\epsilon\left(t, \theta_{l}\right)$ in (23) can be rewritten as follows using (1) and (11):

$$
\begin{aligned}
& \epsilon\left(t, \theta_{l}\right)=e_{l}(t)+\frac{\Delta G_{l}\left(z, \theta_{l}\right)}{H_{l}\left(z, \theta_{l}\right)}\left(R_{l}^{u} r(t)+R_{\text {ext,l }}^{u} r e f_{\text {ext }}(t)+\sum_{i \in \mathcal{L}_{l}} S_{l i}^{u} e_{i}(t)\right) \\
& +\left(\frac{\Delta H_{l}\left(z, \theta_{l}\right)}{H_{l}\left(z, \theta_{l}\right)}+\frac{\Delta G_{l}\left(z, \theta_{l}\right)}{H_{l}\left(z, \theta_{l}\right)} S_{l l}^{u}\right) e_{l}
\end{aligned}
$$


where $\Delta G_{l}\left(z, \theta_{l}\right)=G_{l}\left(z, \theta_{0, l}\right)-G_{l}\left(z, \theta_{l}\right)$ and $\Delta H_{l}\left(z, \theta_{l}\right)=H_{l}\left(z, \theta_{0, l}\right)-H_{l}\left(z, \theta_{l}\right)$. Using now the fact that there is at least a delay in the product $K_{l} G_{l}$ (and thus in the product $\Delta G_{l}\left(z, \theta_{l}\right) S_{l l}^{u}$ ), that $H_{l}$ is monic and that $r$, re $f_{\text {ext }}$ and the white noises $e_{j}\left(j=1, \ldots, N_{\text {mod }}\right)$ are mutually independent, the power $\bar{E} \epsilon^{2}\left(t, \theta_{l}\right)$ of $\epsilon\left(t, \theta_{l}\right)$ is given by:

$$
\bar{E} \epsilon^{2}\left(t, \theta_{l}\right)=\sigma_{e_{l}}^{2}+\bar{E} s_{r}^{2}\left(t, \theta_{l}\right)+\bar{E} s_{r e f_{e x t}}^{2}\left(t, \theta_{l}\right)+\bar{E} s_{e_{l}}^{2}\left(t, \theta_{l}\right)+\sum_{i \in \mathcal{L}_{l}} \bar{E} s_{e_{i}}^{2}\left(t, \theta_{l}\right)
$$

with $s_{r}=\frac{\Delta G_{l}\left(z, \theta_{l}\right)}{H_{l}\left(z, \theta_{l}\right)} R_{l}^{u} r, s_{r e f_{e x t}}=\frac{\Delta G_{l}\left(z, \theta_{l}\right)}{H_{l}\left(z, \theta_{l}\right)} R_{e x t, l}^{u} r e f_{e x t}, s_{e_{l}}=\left(\frac{\Delta H_{l}\left(z, \theta_{l}\right)}{H_{l}\left(z, \theta_{l}\right)}+\frac{\Delta G_{l}\left(z, \theta_{l}\right)}{H_{l}\left(z, \theta_{l}\right)} S_{l l}^{u}\right) e_{l}$ and, for $i \in \mathcal{L}_{l}, s_{e_{i}}=\frac{\Delta G_{l}\left(z, \theta_{l}\right)}{H_{l}\left(z, \theta_{l}\right)} S_{l i}^{u} e_{i}$.

Since $\bar{E} \epsilon^{2}\left(t, \theta_{0, l}\right)=\sigma_{e_{l}}^{2}$, we thus need to have $\bar{E} \epsilon^{2}\left(t, \theta_{l}^{*}\right)=\sigma_{e_{l}}^{2}$ for all minimizers $\theta_{l}^{*}$ of the asymptotic criterion (56). Since we assume that the model structure $\mathcal{M}$ is globally identifiable at $\theta_{0, l}$, the consistency will thus be proven if we show that $\bar{E} s_{r}^{2}\left(t, \theta_{l}\right)=\bar{E} s_{r e f_{e x t}}^{2}\left(t, \theta_{l}\right)=\bar{E} s_{e_{j}}^{2}\left(t, \theta_{l}\right)=0$ $\left(j=l\right.$ and $\left.j \in \mathcal{L}_{l}\right)$ implies $\Delta G_{l}\left(z, \theta_{l}\right)=\Delta H_{l}\left(z, \theta_{l}\right)=0$.

Let us first consider the case where $\mathcal{L}_{l}$ is non-empty i.e. there is at least one path from a node $i \neq l$ to Node $l$. Due to the existence of this path, $S_{l i}^{u}$ is a nonzero transfer function (see Assumption 3). Consequently, $\bar{E} s_{e_{i}}^{2}\left(t, \theta_{l}\right)=0$ implies $\Delta G_{l}\left(z, \theta_{l}\right)=0$. Since $\Delta G_{l}\left(z, \theta_{l}\right)=0$, we have that $\bar{E} s_{e_{l}}^{2}\left(t, \theta_{l}\right)=0$ implies that $\Delta H_{l}\left(z, \theta_{l}\right)=0$ and we have therefore proven the consistency of $\hat{\theta}_{N, l}$ for this case (independently of the value of $r$ and $r e f_{\text {ext }}$ ).

Let us now consider the case where $\mathcal{L}_{l}$ is empty (it is e.g. the case of Node 1 in the network of Figure 1). In that particular case, $y_{r e f, l}$ must $^{9}$ be equal to re $f_{\text {ext }}$. Consequently, the data $\left\{y_{l}(t), u_{l}(t) \mid t=1, \ldots, N\right\}$ are generated by the closed-loop system described by (24)-(25) and the consistency property have to be evaluated on this loop. As shown in e.g. [11], when $\left\{y_{l}(t), u_{l}(t) \mid t=\right.$ $1, \ldots, N\}$ are generated by the closed-loop system described by $(24)-(25)$, the orders of excitation of $r$ and/or re $f_{\text {ext }}$ will need to be sufficient to yield a consistent $\hat{\theta}_{N, l}$.

Finally, let us note that the above reasoning remains exactly the same for the non-stealth setting i.e. when $R_{l}^{u}$ is replaced by $R_{l}^{u, N S}$ in the expression of $s_{r}(t, \theta)$ in (59).

\section{B.2 Accuracy}

Since $\epsilon\left(t, \theta_{0, l}\right)=e_{l}(t)$ and since $\hat{\theta}_{N, l}$ is a consistent estimate of $\theta_{0, l}, \hat{\theta}_{N, l}$ is asymptotically normally distributed around $\theta_{0, l}[22]$ and the inverse of its covariance matrix is given by:

$$
P_{\theta_{l}}^{-1}=\frac{N}{\sigma_{e_{l}}^{2}} \bar{E} \psi_{l}\left(t, \theta_{0, l}\right) \psi_{l}^{T}\left(t, \theta_{0, l}\right)
$$

with $\psi\left(t, \theta_{l}\right)=\frac{-\partial \epsilon\left(t, \theta_{l}\right)}{\partial \theta_{l}}[22]$.

Let us first show that the above expression is equivalent to (26) in the stealth setting. It is easy to show (see e.g. [4]) that $\psi\left(t, \theta_{0, l}\right)=F_{l}\left(z, \theta_{0, l}\right) u_{l}(t)+L_{l}\left(z, \theta_{0, l}\right) e_{l}(t)$ with $F_{l}\left(\theta_{l}\right)$ as defined below (26) and with $L_{l}\left(\theta_{l}\right)=H_{l}^{-1}\left(\theta_{l}\right) \frac{\partial H_{l}\left(\theta_{l}\right)}{\partial \theta_{l}}$.

Using (11) and recalling that $r$ and $r e f_{\text {ext }}$ and the white noises in $\bar{e}$ are all mutually independent, we obtain the expression (26) with [2]:

$$
M_{\bar{e}}=\frac{N}{2 \pi \sigma_{e_{l}}^{2}} \int_{-\pi}^{\pi} \mathcal{Z}_{l}\left(e^{j \omega}\right) \operatorname{diag}\left(\sigma_{e_{1}}^{2}, \sigma_{e_{2}}^{2}, \ldots, \sigma_{e_{N_{m o d}}}^{2}\right) \mathcal{Z}_{l}^{*}\left(e^{j \omega}\right) d \omega
$$

with $\sigma_{e_{i}}^{2}\left(i=1, \ldots, N_{\text {mod }}\right)$ the variance of $e_{i}$ and with $\mathcal{Z}_{l}(z)$ a matrix of transfer functions of dimension $n_{\theta_{l}} \times N_{\text {mod }}$ whose $l^{\text {th }}$ column is $L_{l}+F_{l} S_{l l}^{u}$ and whose $k^{t h}$-column $(k \neq l)$ is equal to $F_{l} S_{l k}^{u}$ $\left(S_{l k}^{u}\right.$ is the entry $l \times k$ of $S^{u}$ in $\left.(11)\right)$.

\footnotetext{
${ }^{9}$ If it was not the case, Node $l$ could not track $r e f_{\text {ext }}$ while we have stated that the objective of the network configuration is that each node follows refext.
} 
In the non-stealth setting, the above reasoning and the expression (26) remain valid, but we have to replace $R_{l}^{u}$ by $R_{l}^{u, N S}$ (see (20)).

\section{Proof of Proposition 3}

Let us introduce the following notations to distinguish the covariance matrice $P_{\theta_{l}}\left(\Phi_{r}, \theta_{0}\right)$ in the stealth and non-stealth case and let us also consider the notations introduced in (54)-(55) to distinguish the individual costs in those two cases.

$$
\begin{aligned}
& P_{\theta_{l}, S}^{-1}\left(\Phi_{r}^{S}, \theta_{0}\right)=M_{\bar{e}}\left(\theta_{0}\right)+ \\
& \frac{N}{2 \pi \sigma_{l}^{2}} \int_{-\pi}^{\pi} F_{l}\left(e^{j \omega}, \theta_{0, l}\right) F_{l}^{*}\left(e^{j \omega}, \theta_{0, l}\right)\left(\left|R_{l}^{u}\left(e^{j \omega}, \theta_{0}\right)\right|^{2} \Phi_{r}^{S}(\omega)+\left|R_{e x t, l}^{u}\left(e^{j \omega}, \theta_{0}\right)\right|^{2} \Phi_{r e f_{e x t}}(\omega)\right) d \omega \\
& P_{\theta_{l}, N S}^{-1}\left(\Phi_{r}^{N S}, \theta_{0}\right)=M_{\bar{e}}\left(\theta_{0}\right)+ \\
& \frac{N}{2 \pi \sigma_{l}^{2}} \int_{-\pi}^{\pi} F_{l}\left(e^{j \omega}, \theta_{0, l}\right) F_{l}^{*}\left(e^{j \omega}, \theta_{0, l}\right)\left(\left|R_{l}^{u, N S}\left(e^{j \omega}, \theta_{0}\right)\right|^{2} \Phi_{r}^{N S}(\omega)+\left|R_{e x t, l}^{u}\left(e^{j \omega}, \theta_{0}\right)\right|^{2} \Phi_{r e f_{e x t}}(\omega)\right) d \omega
\end{aligned}
$$

Using the optimal spectrum $\Phi_{r, o p t}^{N S}$ in the non-stealth case, let us define the following spectrum:

$$
\Phi_{r}^{S}(\omega)=\frac{\left|R_{l}^{u, N S}\left(e^{j \omega}, \theta_{0}\right)\right|^{2}}{\left|R_{l}^{u}\left(e^{j \omega}, \theta_{0}\right)\right|^{2}} \Phi_{r, o p t}^{N S}(\omega)
$$

If an excitation signal $r$ having that spectrum is used during an experiment where the stealth configuration is implemented, it is clear that the obtained covariance matrix $P_{\theta_{l}, S}\left(\Phi_{r}^{S}, \theta_{0}\right)$ will be equal to the one obtained in the non-stealth case with $\Phi_{r, o p t}^{N S}$ (i.e. $\left.P_{\theta_{l}, N S}\left(\Phi_{r, o p t}^{N S}, \theta_{0}\right)\right)$. Consequently, an experiment with this spectrum $\Phi_{r}^{S}$ will satisfy the accuracy constraint (28). Due to (60), (54) and (55), we have also the following relation for the individual cost at Node $l$ :

$$
J_{l}^{S}\left(\Phi_{r}^{S}, \theta_{0}\right)=J_{l}^{N S}\left(\Phi_{r, o p t}^{N S}, \theta_{0}\right)
$$

Moreover, for $i \neq l$, we have that:

$$
\begin{gathered}
\left|R_{i}^{u}\left(e^{j \omega}, \theta_{0}\right)\right|^{2} \Phi_{r}^{S}(\omega)=\left|\bar{N}_{i}\left(e^{j \omega}, \theta_{0}\right)\right|^{2}\left|T_{0, l}\left(e^{j \omega}\right)-T_{i n i t, l}\left(e^{j \omega}\right)\right|^{2} \Phi_{r}^{S}(\omega) \\
\left|R_{i}^{u, N S}\left(e^{j \omega}, \theta_{0}\right)\right|^{2} \Phi_{r, o p t}^{N S}(\omega)=\left|\bar{N}_{i}\left(e^{j \omega}, \theta_{0}\right)\right|^{2}\left|T_{0, l}\left(e^{j \omega}\right)\right|^{2} \frac{\left|R_{l}^{u}\left(e^{j \omega}, \theta_{0}\right)\right|^{2}}{\left|R_{l}^{u, N S}\left(e^{j \omega}, \theta_{0}\right)\right|^{2}} \Phi_{r}^{S}(\omega)
\end{gathered}
$$

Consequently, due to Assumption 1, for $i \in \mathcal{P}_{l}$, we have that $J_{i}^{S}\left(\Phi_{r}^{S}, \theta_{0}\right)<J_{i}^{N S}\left(\Phi_{r, o p t}^{N S}, \theta_{0}\right)$ if $T_{\text {init,l }}$ satisfies (29) for all frequencies $\omega$ where $\Phi_{r}^{S}(\omega) \neq 0$ (and thus for all frequencies $\omega$ where $\left.\Phi_{r, o p t}^{N S}(\omega) \neq 0\right)$. Moreover, for $i \neq l$ and $i \notin \mathcal{P}_{l}$, we have that $J_{i}^{S}\left(\Phi_{r}^{S}, \theta_{0}\right)=J_{i}^{N S}\left(\Phi_{r, o p t}^{N S}, \theta_{0}\right)=0$. Due to (17), we have thus shown that, in the stealth case, we can find a spectrum $\Phi_{r}^{S}$ leading to the same accuracy as with $\Phi_{r, o p t}^{N S}$, but with a strictly smaller cost. The result of the proposition is therefore proven since, by definition, $J^{S}\left(\Phi_{r}^{S}, \theta_{0}\right) \geq J^{S}\left(\Phi_{r, \text { opt }}^{S}, \theta_{0}\right)$ 


\section{Computation of the quantities $\tilde{c}_{i}(\omega)$ and $\rho_{i}(\omega)$}

This appendix shows how the quantities $\tilde{c}_{i}(\omega)$ and $\rho_{i}(\omega)$ (see (48)) can be computed for a given $i$ and a given $\omega$. For this purpose, let us first give an expression of $\tilde{T}_{i}\left(e^{j \omega}, \theta_{i}\right)$ as a function of $\theta_{i}$ using the following notation for $G_{i}\left(e^{j \omega}, \theta_{i}\right)=\frac{Z_{1, i}\left(e^{j \omega}\right) \theta_{i}}{1+Z_{2, i}\left(e^{j \omega}\right) \theta_{i}}$. In the last expression, $Z_{1, i}(z)$ and $Z_{2, i}(z)$ are row vectors containing only delays or zeros (see [1]). This yields

$$
\tilde{T}_{i}\left(e^{j \omega}, \theta_{i}\right)=\frac{-1+Z_{N, i}\left(e^{j \omega}\right) \theta_{i}}{1+Z_{D, i}\left(e^{j \omega}\right) \theta_{i}}
$$

with $Z_{D, i}=Z_{2, i}+K_{i} Z_{1, i}$ and $Z_{N, i}=\frac{Z_{1, i}}{T_{i}\left(e^{j \omega}, \theta_{i n i t, i}\right)}-Z_{D, i}$. Note that, compared to Section 3 in [2], $Z_{N, i}$ and $Z_{D, i}$ have here different expression since $T_{i}$ is defined differently. Based on (61), the quantities $\tilde{c}_{i}(\omega)$ and $\rho_{i}(\omega)$ can be exactly computed using the LMI optimization problem given in the following proposition whose proof can be found in our previous contribution [2].

Proposition 6 Consider the notation $\tilde{T}_{i}\left(e^{j \omega}, \theta_{i}\right)=\frac{-1+Z_{N, i}\left(e^{j \omega}\right) \theta_{i}}{1+Z_{D, i}\left(e^{j \omega}\right) \theta_{i}}$ given in (61). The optimization problem (48) at a given $\omega$ and at a given $i$ is equivalent to the following LMI optimization problem having as decision variables a positive real scalar $\mu_{i}(\omega)$, a complex scalar $\tilde{c}_{i}(\omega)$, a positive real scalar $\xi_{i}(\omega)$ and a skew-symmetric matrix $\mathcal{X}_{i}(\omega) \in \mathbf{R}^{\left(n_{\theta_{i}}+1\right) \times\left(n_{\theta_{i}}+1\right)}\left(n_{\theta_{i}}\right.$ is the dimension of $\left.\theta_{i}\right)$ :

$\min \mu_{i}(\omega)$ subject to

$$
\left(\begin{array}{c|c}
-\mu_{i}(\omega) & \lambda_{i}(\omega) \\
\hline \lambda_{i}^{*}(\omega) & -A_{i}(\omega)-\xi_{i}(\omega) B_{i}+j \mathcal{X}_{i}(\omega)
\end{array}\right)<0
$$

with $j=\sqrt{-1}, \lambda_{i}(\omega)=\left(Z_{N, i}-Z_{D, i} \tilde{c}_{i} \mid-1-\tilde{c}_{i}\right)$ and

$$
A_{i}(\omega)=\left(\begin{array}{cc}
Z_{D, i}^{*} Z_{D, i} & Z_{D, i}^{*} \\
Z_{D, i} & 1
\end{array}\right) \quad B_{i}=\left(\begin{array}{cc}
P_{\text {init }, i}^{-1} & -P_{\text {init }, i}^{-1} \theta_{\text {init }, i} \\
-\theta_{\text {init }, i}^{T} P_{\text {init }, i}^{-1} & \theta_{\text {init }, i}^{T} P_{\text {init }, i}^{-1} \theta_{\text {init }, i}-\chi_{\beta}
\end{array}\right)
$$

The above optimization problem is not explicitly function of $\rho_{i}(\omega)$. However, the optimal $\rho_{i}(\omega)$ can be obtained by taking the square root of the optimal $\mu_{i}(\omega)$.

\section{E Proof of Proposition 5}

The result of Proposition 5 will be proven if we can show that the LMI (52) implies:

$$
\mathcal{F}^{*}\left(M_{\Delta}\left(e^{j \omega}\right), \Delta\left(e^{j \omega}\right)\right) \mathcal{F}\left(M_{\Delta}\left(e^{j \omega}\right), \Delta\left(e^{j \omega}\right)\right)<\tilde{\gamma}(\omega) \quad \forall \Delta\left(e^{j \omega}\right) \in \boldsymbol{\Delta}(\omega)
$$

To show this, let us expand the $\operatorname{LFT} \mathcal{F}\left(M_{\Delta}\left(e^{j \omega}\right), \Delta\left(e^{j \omega}\right)\right)$ into

$$
\bar{p}=\Delta\left(e^{j \omega}\right) \bar{q} \quad \text { and } \quad\left(\begin{array}{c}
\bar{q} \\
\bar{s}
\end{array}\right)=M_{\Delta}\left(e^{j \omega}\right)\left(\begin{array}{c}
\bar{p} \\
1
\end{array}\right)
$$

where $\bar{q}, \bar{p}$ and $\bar{s}$ are complex vectors and where $\bar{s}=\mathcal{F}\left(M_{\Delta}\left(e^{j \omega}\right), \Delta\left(e^{j \omega}\right)\right)$. Let us now consider this LFT (64) for a given $\Delta\left(e^{j \omega}\right) \in \boldsymbol{\Delta}(\omega)$ and let us also consider the corresponding signals $\bar{p}, \bar{q}$ and $\bar{s}$. Let us then pre- and post-multiply the LMI constraint $(52)$ with $\left(\bar{p}^{*}, 1\right)$ and $\left(\bar{p}^{T}, 1\right)^{T}$, respectively. Using (64), this yields:

$$
\left(\begin{array}{c}
\bar{q} \\
\bar{p}
\end{array}\right)^{*} \underbrace{\left(\begin{array}{cc}
T_{\omega}\left(R_{\omega}-C_{\omega}^{*} C_{\omega}\right) & T_{\omega} C_{\omega}^{*} \\
T_{\omega} C_{\omega} & -T_{\omega}
\end{array}\right)}_{=A}\left(\begin{array}{c}
\bar{q} \\
\bar{p}
\end{array}\right)+\bar{s}^{*} \bar{s}<\tilde{\gamma}(\omega)
$$


Since $\bar{p}=\Delta\left(e^{j \omega}\right) \bar{q}$, we can rewrite the first term of the left hand side of (65) as follows:

$$
\left(\begin{array}{c}
\bar{q} \\
\bar{p}
\end{array}\right)^{*} A\left(\begin{array}{c}
\bar{q} \\
\bar{p}
\end{array}\right)=\bar{q}^{*}\left(\begin{array}{c}
I_{N_{m o d}} \\
\Delta\left(e^{j \omega}\right)
\end{array}\right)^{*} A\left(\begin{array}{c}
I_{N_{m o d}} \\
\Delta\left(e^{j \omega}\right)
\end{array}\right) \bar{q}
$$

The above reasoning can be done for any value of $\Delta\left(e^{j \omega}\right) \in \boldsymbol{\Delta}(\omega)$. In other words, for the matrix $T_{\omega}$ found by the optimization problem, (65) holds true for all $\Delta\left(e^{j \omega}\right) \in \boldsymbol{\Delta}(\omega)$. Let us also observe that, when $T_{\omega}$ is a strictly positive definite diagonal matrix, (66) is a positive quantity for all $\Delta\left(e^{j \omega}\right) \in \boldsymbol{\Delta}(\omega)$. Recalling that $\bar{s}=\mathcal{F}\left(M_{\Delta}\left(e^{j \omega}\right), \Delta\left(e^{j \omega}\right)\right)$, we have therefore also that (63) holds, which is the desired result. 